\title{
A network description of the non-Gaussian stress-optic and Raman scattering responses of elastomer networks
}

\author{
P. R. von Lockette and E. M. Arruda, Ann Arbor, Michigan
}

(Received November 14, 1997)

\begin{abstract}
Summary. The ability to measure orientation in dual or multi-phase materials is of current relevance in the study of the constitution and deformation characteristics of the separate phases in many technologically important polymeric systems. Raman spectroscopy is a very useful tool in this regard because separation of the scattered Raman intensities by phase is possible and because it can be used accurately on thick specimens. A three dimensional network model concept used previously to describe the stress and birefringence reponses of elastomers is extended to describe the components of the Raman tensor for amorphous elastomers under general finite deformations. The utility of the model is verified via its ability to predict the finite deformation responses of elastomeric networks under large shear deformations. Polydimethylsiloxane (PDMS) networks are tested to large deformations in uniaxial compression and in shear for comparison with the theory. Simultaneous displacement, load and optical retardation data are collected using apparatus specially designed to allow optical access throughout the deformation tests. The importance of properly accounting for finite rotations when relating the computational results to the experimentally measured optical data is discussed. The proposed network description of the Raman tensor is also compared to Raman intensity in the literature on polyethylene terephthalate (PET). The results indicate that the theory accurately predicts the anisotropic Raman tensor components over the full range of deformation for which data are available.
\end{abstract}

\section{Background}

It is well known that under an applied deformation a polymer develops mechanical and optical anisotropy although characterization of the anisotropic stress-optic response of multiphase polymeric networks at large deformations is still under consideration. Birefringence, or the difference in refractive indices in two orthogonal directions in an anisotropic medium, is a measure of optical anisotropy that provides an accurate description of orientation for deformations in which the Gaussian approximation holds. Birefringence is readily measured for amorphous polymers under Gaussian deformations, and the resulting linear stress-optic behavior is exploited in photoelasticity [1] and in flow visualization techniques [2], [3] to deduce components of the stress tensor. Characterization of orientation in the non-Gaussian deformation range is of current interest in the study of polymer flows [4], [5], amorphous and semicrystalline polymers [6], and in elastomers [7]-[10].

With regard to multi-phase materials such as semi-crystalline polymers, an orientation measurement technique whose signal is separable is desirable. For large deformations Raman spectroscopy can provide a thorough description of orientation for fairly smooth orientation distribution functions of scattering units [11]. Moreover, the contributions to the total Raman 
scattered intensities due to the crystal lattices and amorphous regions in semi-crystalline polymers have been determined in several instances [6], [12], [13]. The use of Raman spectroscopy has been limited to describing orientation by identifying a scattering unit with a particular Raman line and relating the intensity of the Raman signal at that line to an orientation distribution function for the scattering unit. The analysis used to relate Raman spectroscopy data to orientation via the spherical harmonics of the orientation distribution function of the scattering units is due to Bower [14], [15]. This procedure has been used to describe orientation in amorphous and semi-crystalline polymers under uniaxial tension by many researchers [6], [12], [13], [16] - [18]. The components of scattered Raman intensity have not previously been related to the deformation of the polymer network which contains the scattering units to predict Raman intensity data versus finite deformation. Moreover, recent studies on semi-crystalline polypropylene have demonstrated that the deformation of the amorphous and crystalline phases in spherulites is non-homogeneous [19], and varies from tension to shear under globally imposed uniaxial tension [20]. Polycrystalline constitutive models of semi-crystalline polypropylene which account for the non-homogeneities associated with the deformation [21] are currently limited by a lack of information about the constitution of the amorphous phase [22]-[24] and its large deformation response. A measure of orientation in the amorphous regions and a means to relate it directly to the deformation of the polymeric chains comprising this region would be very useful in the characterization of the amorphous deformation response. Similar information about the evolution of texture in the crystals is accessed through X-ray diffraction, and related to the orientation of crystallographic slip planes in polymer crystal plasticity models [25].

A network model capable of predicting scattered Raman intensities for an amorphous polymer under a general deformation state is sought. In some instances of interest local deformation and orientation may be quite large thus the model should consider non-Gaussian chain statistics. The scattered Raman tensor intensities and the deformation of a polymeric chain network may be related through the network polarizability. Birefringence is similarly related to deformation via polarizability, thus a network theory that predicts the birefringence response for a generalized deformation state would also predict the scattered Raman intensities. We first briefly discuss existing birefringence network theories to further motivate the utility of a Raman network theory, and then propose a network theory for the Raman scattered intensities of an amorphous polymer.

\subsection{Birefringence theories}

The stress and optical responses of elastomer chains and the networks they form have generally been treated considering affine deformation of the network chains using Gaussian statistics in the familiar works of several authors [26]- [29]. The statistical theory of rubber elasticity yields for a Gaussian [26] network in uniaxial tension the stress response

$\sigma=n k \Theta\left(\lambda^{2}-\frac{1}{\lambda}\right)$

where $\sigma$ is the true stress, $n$ is the chain density, $k$ is Boltzmann's constant, $\Theta$ is temperature, and $\lambda$ is the applied stretch. Similarly, the Gaussian network theory describes the optical anisotropy in uniaxial tension in terms of the stretch as

$\Delta \eta_{1-2}=\frac{2 \pi \alpha n}{45} \frac{\left(\eta_{0}^{2}+2\right)^{2}}{\eta_{0}}\left(\lambda^{2}-\frac{1}{\lambda}\right)$ 
where $\Delta \eta_{1-2}$ is a principal birefringence, more specifically the difference in principal refractive indices in the plane normal to the third principal axis, $\alpha$ is the optical anisotropy of a statistical segment, and $\eta_{0}$ is the mean refractive index of the medium. The familiar stress-optic law results from combining rubber elasticity and rubber birefringence theories for Gaussian chain deformation

$\Delta \eta_{1-2}=C \sigma ; \quad C=\frac{2 \pi \alpha}{45 k \Theta} \frac{\left(\eta_{0}^{2}+2\right)^{2}}{\eta_{0}}$

where $C$ is the stress-optic coefficient.

More recently non-Gaussian stress-optic behavior in polymers and elastomers has been investigated by a number of authors. Non-Gaussian chain statistics has been used to describe the stress and optical responses of steady and unsteady polymer flows via the Doi Edwards model with segmental stretch [4], [5]. Investigations are currently underway to deduce the segmental orientation from scattering experiments on elongational flows in an effort to probe the segmental stretch dynamics [30]. Experimental results depicting nonlinear stress-optic behavior in PDMS polymers in uniaxial tension [7], [8] have renewed interest in characterizing and modelling the stress optic response of elastomers at large deformations.

Non-Gaussian affine network theories of the stress response of elastomers were originally developed [26], [27] to describe rubbery deformations approaching limiting chain extensibility. These theories were recently shown to perform inadequately as three-dimensional constitutive laws for rubbery materials [31]. Rubbery orientation theories based on those same network geometries would similarly be expected to inadequately predict either the birefringence or Raman scattering of an elastomer under general three-dimensional stretch-states [9].

Recently, a non-Gaussian rubber birefringence theory based on the eight-chain network [31] demonstrated an ability to predict both birefringence and stress responses of elastomeric networks in uniaxial tension based on characterization using uniaxial compression and a trio of independent, physically-based parameters [9]. The eight-chain model of rubber elasticity gives

$\sigma_{i}-\sigma_{j}=\frac{n k \Theta}{3} \sqrt{N} \mathcal{L}^{-1}\left\{\frac{\lambda_{c h}}{\sqrt{N}}\right\} \frac{\lambda_{i}{ }^{2}-\lambda_{j}{ }^{2}}{\lambda_{c h}}$

where $i$ and $j$ are indexes for principal direction, $N$ is the number of statistical segments per chain, $\lambda_{c h}=\frac{1}{\sqrt{3}}\left(\lambda_{1}{ }^{2}+\lambda_{2}{ }^{2}+\lambda_{2}{ }^{2}\right)^{1 / 2}$ is the stretch on each chain in the network, $\lambda_{1}, \lambda_{2}$, and $\lambda_{3}$ are the applied principal stretches, and $\mathcal{L}^{-1}\left\{\frac{\lambda_{c h}}{\sqrt{N}}\right\}$ is the inverse Langevin function given by $\mathcal{L}\{\chi\}=\operatorname{coth} \chi-\frac{1}{\chi}$

The non-Gaussian network birefringence model gives the fully three-dimensional birefringence versus stretch reponse as [9]

$\Delta \eta_{i j}=\frac{2 \pi}{9} \frac{\left(\eta_{0}{ }^{2}+2\right)^{2}}{\eta_{0}} \frac{n \alpha N}{3 \lambda_{c h}^{2}}\left(1-\frac{\frac{3 \lambda_{c h}}{\sqrt{N}}}{\mathcal{L}^{-1}\left\{\frac{3 \lambda_{c h}}{\sqrt{N}}\right\}}\right)\left(\lambda_{i}{ }^{2}-\lambda_{j}{ }^{2}\right)$.

The response of this model is characterized by only three independent physically-based parameters, $n, N$, and $\alpha$, the chain link density, number of links per chain, and optical anisotropy 
in a statistical segment, respectively. At small deformations (i.e. Gaussian deformation ranges) Eqs. (4) and (6) reduce to the stress-optic law of Eq. (3) [9].

Wu and van der Giessen [10] recently developed a non-Gaussian network description of orientation based on the full network model of Treloar [29] and compared birefringence simulations to predictions of other non-Gaussian models [9], [29] in tension and simple shear. None of these existing non-Gaussian models of orientation has demonstrated the ability to predict experimental optical anisotropy results in non-homogeneous deformation states such as the large deformation shear test. This is a necessary step in validating a network based birefringence or Raman spectroscopy model. Since birefringence is related to the Raman tensor through the first spherical harmonic of the orientation distribution function, and because the retardation measured in a birefringence test is straightforward to interpret, birefringence will be used here to validate the network orientation modelling approach for general deformations via large deformation shear tests in Section 3.

\subsection{Raman spectroscopy}

Raman spectroscopy also allows for analysis of the orientation of polymeric and elastomeric materials. Raman scattering occurs when polarized light incident on a sample excites a particular vibrational mode of a certain molecular species. Raman scattering is spectral; the frequency of incident light determines the molecular species and the vibrational mode excited. The intensity of the scattered light depends on the orientation of the molecular species, the orientation of the incident and scattered light, and the particular vibrational mode investigated. The orientation of the principal axes of that vibrational mode can then be analyzed through a set of experiments carried out at the excited frequency. The intensity of scattered light from Raman experiments $I_{s}$ is determined by

$I_{s}=\sum_{\text {scatterers }} \sum_{i j}\left(l_{i} l_{j}^{\prime} \alpha_{i j}^{\prime}\right)^{2}$

where $l_{i}$ is the direction cosine of the polarization vector of the incident light with respect to the specimen axes, and $l_{j}{ }^{\prime}$ is the direction cosine of the allowed polarization vector of an analyzer with respect to the specimen axes. The expression in Eq. (7) is quadratic in $\alpha_{i j}^{\prime}$, the polarizability tensor of the vibrational mode in question; knowledge of the polarizability tensor components is necessary to quantitatively predict results of a Raman scattering experiment.

In the analysis of Bower [14], [15] the polarizability tensor was computed by considering a distribution of scattering units. For a specimen with uniaxial symmetry the scattered Raman intensities are described in the Bower analysis as functions of the Legendre polynomials $\left\langle P_{l}(\cos \theta)\right\rangle$ of the orientation distribution function and the principal polarizabilities of the vibration investigated. This elegant analysis is often used to describe orientation in uniaxially oriented specimens by using the experimentally determined Raman scattered intensities to compute the second and fourth Legendre polynomials, $\left\langle P_{2}(\cos \theta)\right\rangle$ and $\left\langle P_{4}(\cos \theta)\right\rangle$. The Bower method, however, treats each scatterer as a separate entity, unconnected to other portions of the system. It does not account for the connectedness of polymeric systems either at the molecular (chain) or the network level. Thus the Bower method may not be used to predict orientation given by the deformation, only quantify it in terms of the coefficients $P_{2}$ and $P_{4}$ from scattered intensity data.

Recently Mead [30] has developed an expression for the Raman tensor based upon a Kuhn and Grün averaging analysis of the freely jointed chain model [29]. The transition from a single 
chain to all chains in the network is performed by multiplying the result for a single chain by the chain density. This approach incorporates connectedness of the links in a freely jointed chain, and assumes the result for the single chain provides the Raman tensor of the average chain.

In the next Section an expression for the scattered Raman intensities $I_{s}$ in terms of the principal network stretches is developed based on a non-Gaussian network description of polymer chain orientation. The freely jointed chain model is used in conjunction with a geometric contraction of the network to determine Raman tensor quantities while incorporating the effects of a proposed network topology. The model is valid for the general deformation state and can be used to predict the Raman intensities that are accessible directly from experimentation. The theory is then compared to Gaussian and single chain non-Gaussian theories for the Raman intensities.

In Sections 3 to 6 we investigate the capability of the theory to predict the experimentally obtained optical anisotropy of PDMS under a large shear deformation, which takes into account the complex, localized inhomogeneity of the deformation and large rotations of the principal stretch directions. Since birefringence is related to Raman through the orientation distribution function and birefringence experiments are straightforward to conduct an interpret, birefringence is used here to validate the network model description of orientation under a complicated deformation state. We show with the numerical simulations the proper procedures for relating the numerically determined retardation to experimental results. This result marks the first successful attempt to predict the global load and retardation responses of elastomers in large, inhomogeneous deformation states in which the principal stretch directions rotate with the deformation.

In Section 6 Raman scattering data from the literature are examined to determine the ability of the network theory to predict scattered Raman intensities versus deformation. Data from Purvis and Bower [17] on PET uniaxially drawn in the rubbery regime are used. We demonstrate that the network theory model is capable of predicting the Raman scattered intensities due to a symmetric stretching vibration quite accurately.

\section{A network description of the Raman tensor}

The freely jointed chain model describes the actual polymer chain in terms of a number of statistical rigid links. Each link represents several monomers (repeat units). In this discussion polarizabilities are designated $\alpha^{\prime}$ for a repeat unit, $\alpha$ for a link, $\gamma$ for a chain and $\beta$ for a complete network. Subscripts $i j$ denote tensor position components, $x y$ denote tensor values in the $x y$ reference directions, and $\|$ and $L$ denote components parallel and perpendicular to the unit, link, or chain in question.

Raman spectroscopy is used to examine any number of vibrational modes associated with the bonds of a polyatomic molecule. Any of these vibrations may be examined for purposes of developing a network polarizability model. Here, axial stretching of backbone bonds is chosen which leads to a form for the network polarizability that is valid for a transversely isotropic link polarizability. This scattering unit has principal polarizabilities $\alpha_{1}{ }^{\prime}, \alpha_{2}{ }^{\prime}, \alpha_{3}{ }^{\prime}$ along the bond axes. In the network theory connectedness among the scattering units is incorporated through the statistical links of the freely jointed chain model. The chains are connected in turn into a polymeric network. In the absence of large side groups $\alpha_{1}{ }^{\prime}, \alpha_{2}{ }^{\prime}, \alpha_{3}{ }^{\prime}$ are analogous to repeat unit polarizabilities [6] and may be used to determine the link polarizabilities $\alpha_{1}, \alpha_{2}$, and $\alpha_{3}$ [32]. For an axially symmetric stretching mode it is assumed that the polariz- 
ability of the rigid link is transversely isotropic; $\alpha_{1}=\alpha_{\|}$along the link, and $\alpha_{2}=\alpha_{3}=\alpha_{\perp}$ perpendicular to the link.

With the axial and transverse link polarizabilities given, the polarizability tensor for a link whose chain end-to-end vector lies along the $0 x$ axis is given by [29]

$\alpha_{x x}=\alpha_{\|} \cos ^{2} \theta+\alpha_{\perp} \sin ^{2} \theta$

$\alpha_{y y}=\left(\alpha_{\|}-\alpha_{\perp}\right) \sin ^{2} \theta \cos ^{2} \phi+\alpha_{\perp}$

$\alpha_{z z}=\left(\alpha_{\|}-\alpha_{\perp}\right) \sin ^{2} \theta \sin ^{2} \phi+\alpha_{\perp}$

$\alpha_{x z}=\alpha_{x y}=\left(\alpha_{\|}-\alpha_{\perp}\right) \sin \theta \cos \theta \cos \phi$

$\alpha_{y z}=\alpha_{y x}=\left(\alpha_{\|}-\alpha_{\perp}\right) \sin ^{2} \theta \sin \phi \cos \phi$

$\alpha_{x z}=\alpha_{z x}=\left(\alpha_{\|}-\alpha_{\perp}\right) \sin \theta \cos \theta \sin \phi$

where $\theta$ is the angle the link makes with the $0 x$ axis and $\phi$ is the angle in the $y z$ plane. Integration over the orientation distribution function for the distribution of link angles with respect to the chain axis yields the polarizability tensor of the chain [29]

$\gamma_{x x}=N\left(\alpha_{\|}-2\left(\alpha_{\|}-\alpha_{\perp}\right) Q\right)$

$\gamma_{y y}=\gamma_{z z}=N\left(\alpha_{\perp}+\left(\alpha_{\|}-\alpha_{\perp}\right) Q\right)$

where

$Q=\frac{\frac{r}{r_{\max }}}{\mathcal{L}^{-1}\left(\frac{r}{r_{\max }}\right)}$.

The term $Q$ represents the strength of the link anisotropy due to stretch in the chain in which $r$ is the current chain length, $r_{\max }$ is the fully extended chain length, and $\mathcal{L}^{-1}$ is the inverse Langevin function. Using a root-mean-square assumption for the initial chain length, $r_{o}=\sqrt{N} l$, where $l$ is the length of a rigid link and $N$ is the number of rigid links in a chain, and assuming affine deformation, $r=\lambda_{c h} r_{o}$ where $\lambda_{c h}$ is the stretch in a chain, yields

$Q=\frac{\frac{\lambda_{c h}}{\sqrt{N}}}{\mathcal{L}^{-1}\left(\frac{\lambda_{c h}}{\sqrt{N}}\right)}$.

The inverse Langevin may be represented in terms of the series expansion

$\mathcal{L}^{-1}(\chi)=3 \chi+\frac{9}{5} \chi^{3}+\frac{297}{175} \chi^{5}+\frac{1539}{875} \chi^{7}+\frac{126117}{67375} \chi^{9}+\cdots$

It is necessary to determine the combined polarizability tensor of an ensemble of chains to describe the three dimensional network response. To do so one must describe the orientations of each of the chains with respect to a fixed set of axes. The eight chain model [31] provides a basis for predicting the results of a Raman scattering experiment by providing a physical network representation from which a polarizability tensor may be generated [9]. Each of the chains in the eight-chain model contributes, in a tensor summation fashion, to the polarizability of the network to yield

$\beta_{i j}=n N\left(\left(\alpha_{\|}-\alpha_{\perp}\right)(1-3 Q)\left(\frac{\lambda_{i}}{\lambda_{c h}}\right)^{2}+\left(\alpha_{\perp}+\left(\alpha_{\|}-\alpha_{\perp}\right) Q\right)\right) \delta_{i j}$

where $i, j=1,2,3, \delta_{i j}$ is the Kronecker delta function, and $\lambda_{c h}^{2}=\frac{1}{3}\left(\lambda_{1}{ }^{2}+\lambda_{2}{ }^{2}+\lambda_{3}{ }^{2}\right)$. 
The network polarizability expression of Eq. (13) provides the principal polarizability tensor in terms of the principal stretches $\lambda_{1}, \lambda_{2}, \lambda_{3}$ from which the Raman tensor can be calculated. Under general deformation states involving rotations of the principal stretch directions, the principal stretch tensor $\hat{\mathbf{V}}$ may be found from an eigenvalue decomposition of the applied stretch tensor V. Equation (13) yields the principal polarizability tensor and Eq. (7) gives the Raman scattered intensity in a given experiment for incident light polarization $l_{i}$ and analyzer polarization $l_{j}^{\prime}$. For deformations much less than the limiting extensibility of the network chains, the polarizability tensor of a Gaussian network may be used to compare Raman scattering predictions. The principal polarizabilities of the Gaussian network under a uniaxial stretch $\lambda$ along the 3 direction are given by [29]

$$
\begin{aligned}
& \beta_{33}=\frac{1}{3} N\left(\alpha_{\|}+2 \alpha_{\perp}\right)+\frac{2}{15}\left(\alpha_{\|}-\alpha_{\perp}\right)\left(\lambda^{2}-\frac{1}{\lambda}\right) \\
& \beta_{11}=\beta_{22}=\frac{1}{3} N\left(\alpha_{\|}+2 \alpha_{\perp}\right)-\frac{1}{15}\left(\alpha_{\|}-\alpha_{\perp}\right)\left(\lambda^{2}-\frac{1}{\lambda}\right)
\end{aligned}
$$

Uniaxial tension is the most commonly used deformation state for characterizing orientation via Raman spectroscopy. In uniaxial tension along the 3 direction there are five independent components of the Raman tensor: $\sum\left(\alpha_{33}^{\prime}\right)^{2}, \sum\left(\alpha_{22}^{\prime}\right)^{2}, \sum\left(\alpha_{23}^{\prime}\right)^{2}, \sum\left(\alpha_{12}^{\prime}\right)^{2}$, and $\sum\left(\alpha_{22}^{\prime} \alpha_{33}^{\prime}\right)$. The first four of these intensities are accessed through right angle or backscattering experiments, but $\sum\left(\alpha_{22}^{\prime} \alpha_{33}^{\prime}\right)^{2}$ is not directly measured using either technique and is instead usually computed through oversampling of the other intensities (see for example [6]). For uniaxial deformation the network theory predicts that the Raman tensor components $\sum\left(\alpha_{12}^{\prime}\right)^{2}$ and $\sum\left(\alpha_{23}^{\prime}\right)^{2}$ due to a symmetric backbone stretching mode are zero. This is a consequence of using a contracted network model in lieu of averaging over all network chain orientations. In future work the authors show how additional chains in a contracted network serve to provide non-zero magnitudes for the off-diagonal components of the Raman tensor without appreciably changing the magnitudes of the diagonal terms [33]. It is expected that in reality these components will range about an order of magnitude less than the other independent components. The predicted intensities of the remaining components of the Raman tensor may be normalized by $n \alpha_{\|}$and plotted versus axial stretch $\lambda$ if values of the network chain limiting extensibility, $\left(\lambda_{c h}\right)_{L}=\sqrt{N}$, and link polarizability ratio, $\frac{\alpha_{\perp}}{\alpha_{\|}}$, are
known.

A backscattering geometry with incident laser and analyzer polarizations aligned with the 3 direction yields $I_{s}=\sum\left(\alpha_{33}^{\prime}\right)^{2}$. Figure 1 contains network theory predictions for normalized Raman scattered intensity along the axial stretch direction, $\frac{\left(\beta_{33}\right)^{2}}{n \alpha_{\|}}$, versus axial stretch $\lambda_{i} ;\left(\beta_{33}\right)^{2}$ is the network theory prediction corresponding to the experimentally measured intensity $\sum\left(\alpha_{33}^{\prime}\right)^{2}$. A link polarizability ratio of $\frac{\alpha_{\perp}}{\alpha_{\|}}=-0.18$ and limiting extensibility of $\left(\lambda_{c h}\right)_{L}$ $=\sqrt{N}=4.48$ were assumed ${ }^{1}$ to simulate uniaxial tension and compression in the range $0.1=\lambda=7.75$. The tension simulations show an increase in scatterer alignment and hence polarizability along the 3 direction which eventually saturates as the network approaches its full extensibility. In compression the intensity drops off sharply as the scattering elements under investigation rotate away from the stretching axis. We also see the beginnings of the

\footnotetext{
1 These values were chosen to simulate polyethylene terephthalate; the details involved are discussed in Section 6.
} 


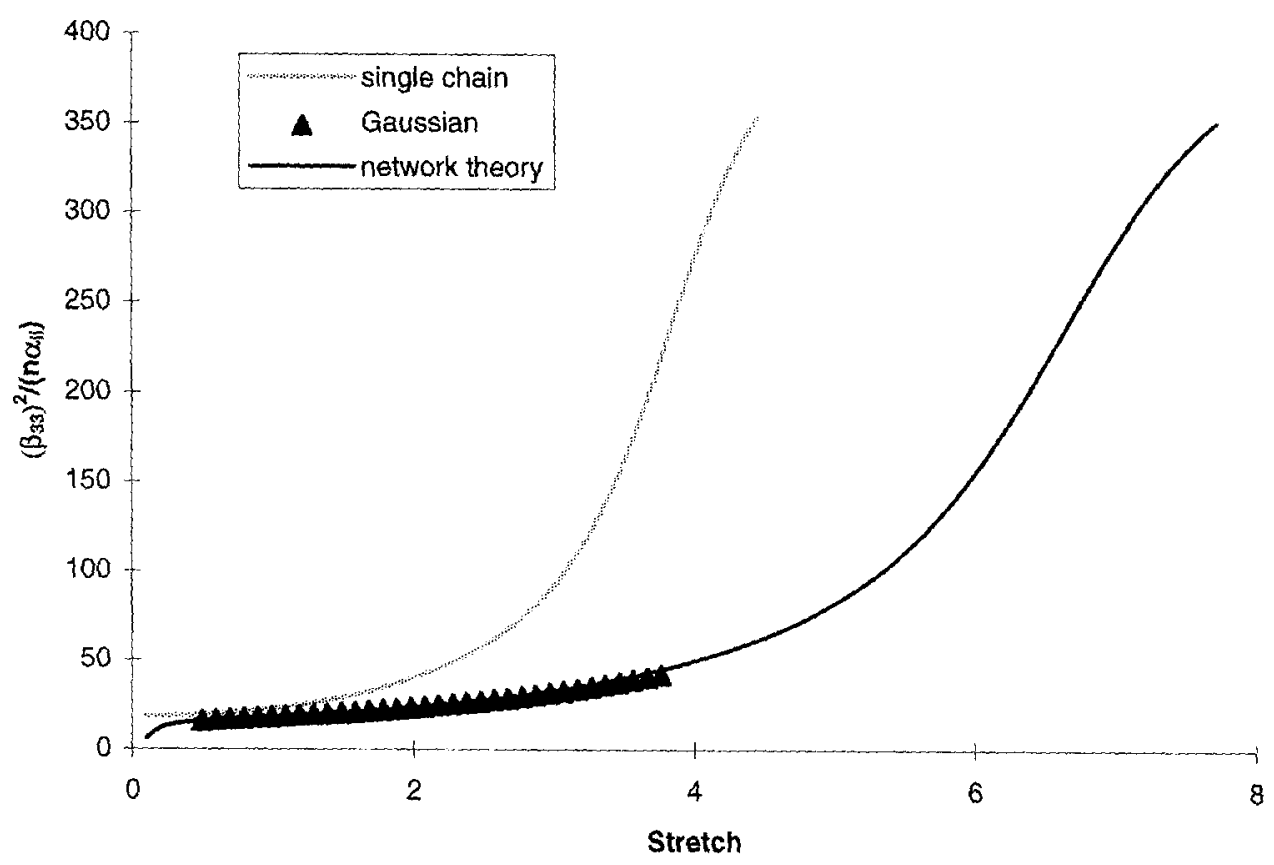

Fig. 1. Normalized Raman scattering simulations for $\frac{\left(\beta_{33}\right)^{2}}{n \alpha_{14}}$ along the uniaxial stretch direction from the network theory, single non-Gaussian chain and Gaussian network

incompressibility effect which forces material expansion, and thereby chain stretch, perpendicular to the axis of compression. The normalized $\frac{\left(\beta_{33}\right)^{2}}{n \alpha_{\|}}$intensity simulation using the single non-Gaussian chain of Eq. (9) is also included in Fig. 1 for comparison with the network theory simulations. For the single chain of Eq. (9) aligned along the stretching direction, $\left(\beta_{x x}\right)^{2}=\left(\beta_{33}\right)^{2}$ and $\left(\beta_{y y}\right)^{2},\left(\beta_{z z}\right)^{2}=\left(\beta_{11}\right)^{2},\left(\beta_{22}\right)^{2}$. The single chain intensity $I_{s}=\frac{\left(\beta_{33}\right)^{2}}{n \alpha_{4}}$ is also simulated for $\frac{\alpha_{\perp}}{\alpha_{\|}}=-0.18$ and $\left(\lambda_{c h}\right)_{L}=\sqrt{N}=4.48$. As the single chain stretches in tension the intensity along the stretch direction increases rapidly until an eventual saturation at an applied stretch equal to the chain locking stretch of $\lambda=\left(\lambda_{c h}\right)_{L}=4.48$. In contrast locking is delayed to an applied stretch of $\lambda=7.75$ due to the network response of the theory (13); in the network theory the chains are not aligned with the principal stretch axes. In compression the ends of the single chain collapse about a point yielding a mean polarizability, i.e. $\frac{N}{3}\left(\alpha_{\|}+2 \alpha_{\perp}\right)$. Simulations of $I_{s}=\frac{\left(\beta_{33}\right)^{2}}{n \alpha_{\|}}$for the Gaussian network using Eqs. (14) and (15) in (7) also appear in Fig. 1 to illustrate that the network theory reduces to the Gaussian theory at small stretches.

The component of the polarizability tensor normal to the uniaxial stretching direction is accessed through backscattering with the incident laser and analyzer polarizations aligned along the 2 axis to yield $I_{s}=\frac{\left(\beta_{z z}\right)^{2}}{n \alpha_{y}}$. Simulations using the network theory, single nonGaussian chain, and Gaussian network appear in Fig. 2 for $\frac{\alpha_{1}}{\alpha_{\|}}=-0.18$ and $\left(\lambda_{c h}\right)_{L}=\sqrt{N}$ $=4.48$. The network theory simulations show that the normalized polarizability normal to the uniaxial stretching direction drops steadily in tension as the chains stretch normal to the 


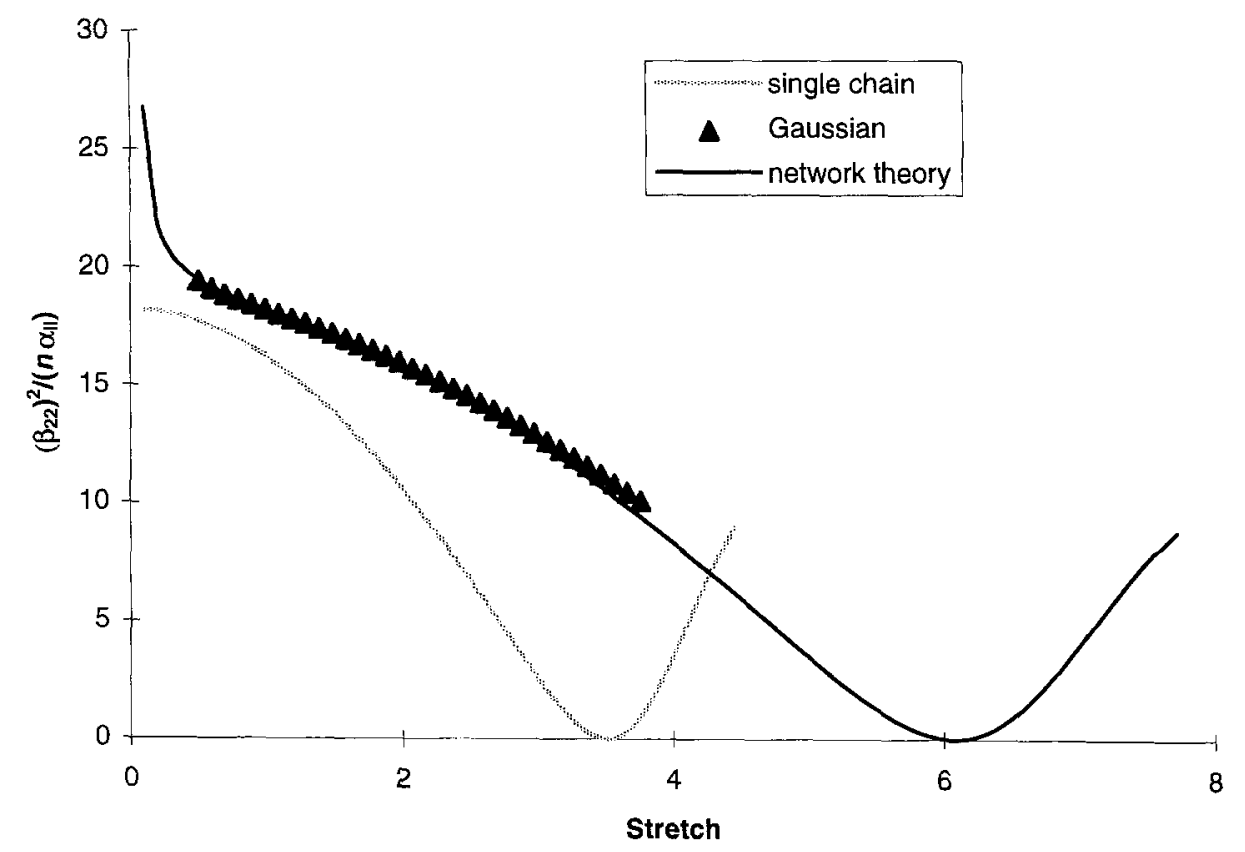

Fig. 2. Normalized Raman scattering simulations for $\frac{\left(\beta_{22}\right)^{2}}{n \alpha}$ normal to the uniaxial stretch direction from the network theory, single non-Gaussian chain and Gaussian network

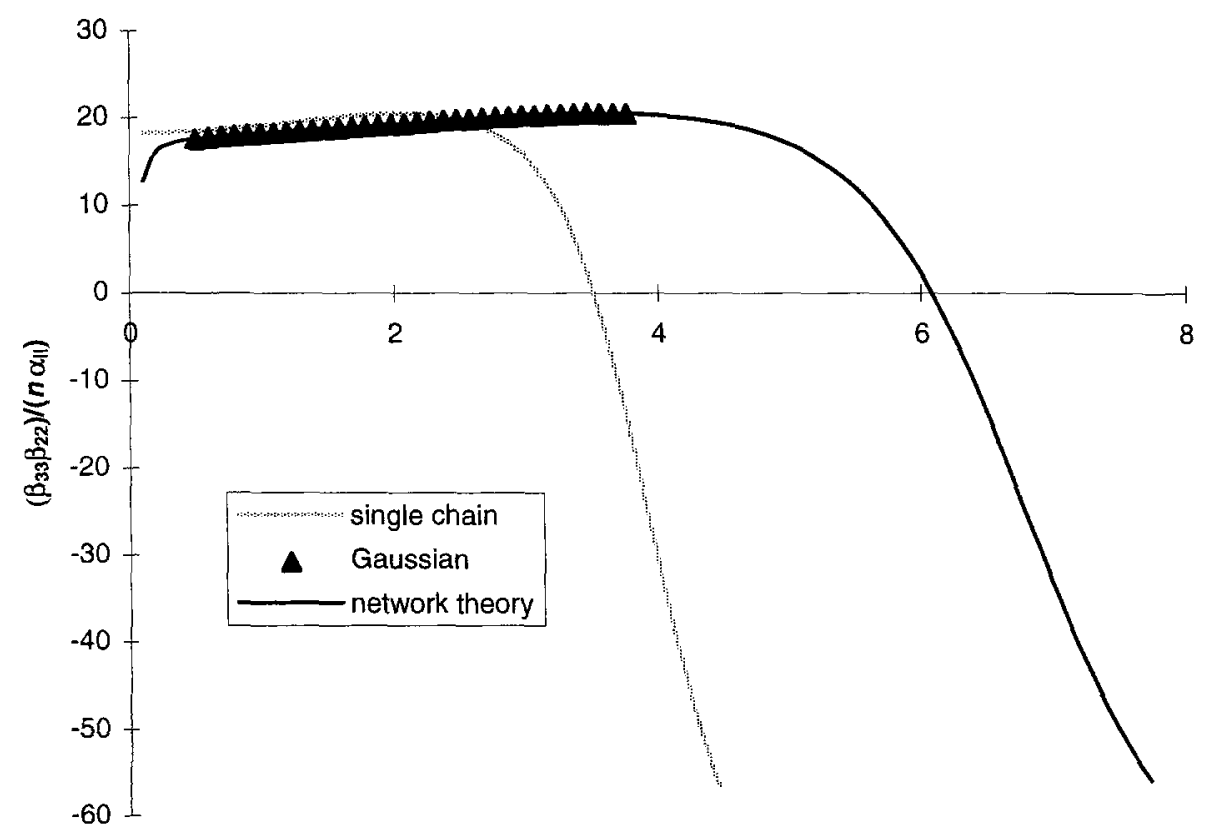

Stretch

Fig. 3. Normalized Raman scattering simulations for the $\frac{\left(\beta_{22} \beta_{33}\right)}{n \alpha_{\|}}$component of the Raman tensor from the network theory, single non-Gaussian chain and Gaussian network 
transverse direction for these scatterers. From (13), this polarizability will equal zero when

$(1-3 Q)\left(\frac{\lambda_{2}}{\lambda_{c h}}\right)^{2}+Q=-\frac{\alpha_{\perp}}{\alpha_{\|}-\alpha_{\perp}}$.

The intensity then increases as the chains approach their limiting extensibility due to the anisotropic contribution to the polarizability, the first term in (13). As in Fig. 1, limiting extensibility is seen to occur at a larger applied stretch for the network theory than for the single chain. In compression the network theory predicts this intensity component increases sharply as chains, and the scatterers under investigation which are linked to the chains, align normal to the compression axis and stretch due to incompressibility. The intensity simulation using the single non-Gaussian chain predicts only a modest increase in scattering intensity as the single chain compresses normal to the polarizability component being probed. Again, the ends of the single chain collapse to a point yielding a mean polarizability $\beta_{11}=\beta_{22}=\beta_{33}=\frac{N}{3}\left(\alpha_{\|}+2 \alpha_{\perp}\right)$. The Gaussian simulation is included to again illustrate that the network theory reduces to the Gaussian theory at small stretches.

Figure 3 contains simulations of the $\frac{\left(\beta_{22} \beta_{33}\right)}{n \alpha_{\|}}$component of the Raman tensor which is not directly measured in spectroscopy experiments [6], but nonetheless represents an independent component of the Raman tensor in uniaxial deformation. This intensity component is fairly insensitive to stretch until very large stretches approaching the limiting extensibility of the network.

In Fig. 4 the ratio of the two Raman tensor components, $\frac{\left(\beta_{22}\right)^{2}}{\left(\beta_{33}\right)^{2}}$, is plotted versus stretch for the network theory, single non-Gaussian chain and Gaussian network. The intensity of this component drops steadily with increasing stretch in tension and rises sharply with compressive stretch. The network theory prediction again superposes accurately onto the Gaussian predic-

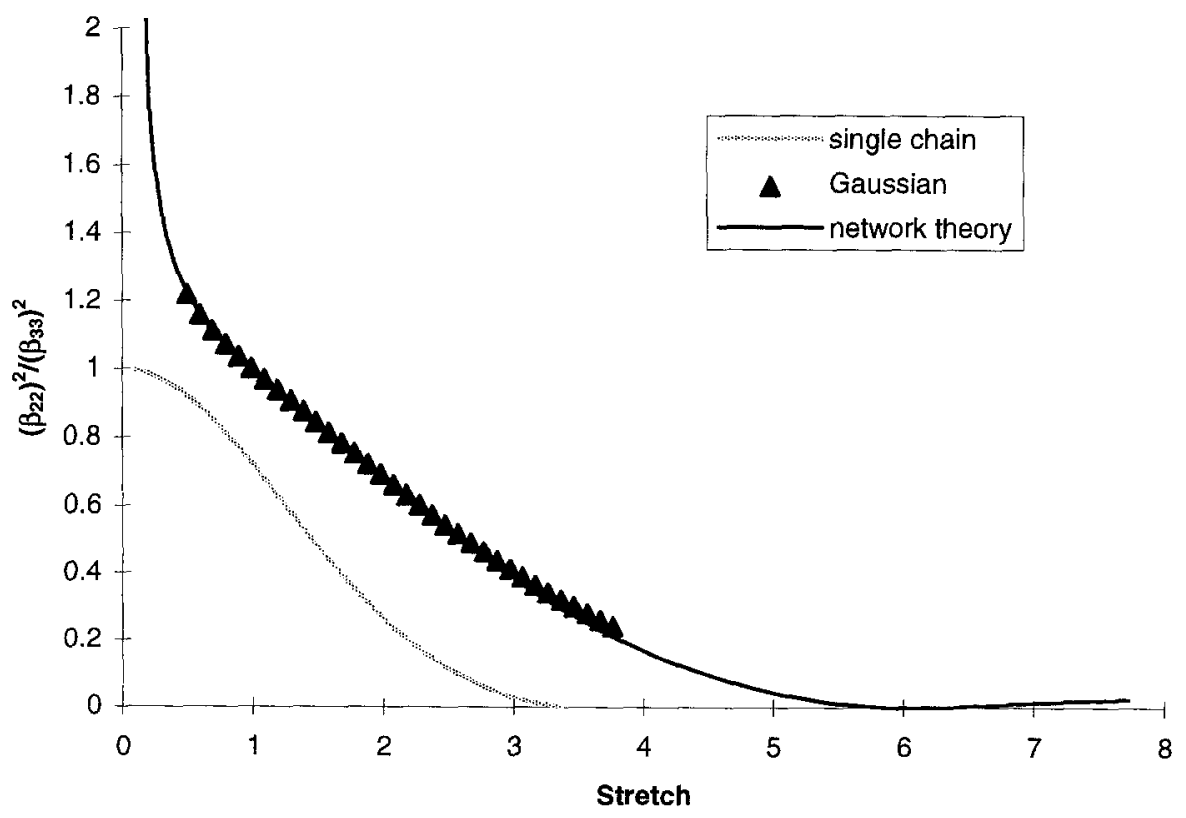
Fig. 4. Simulations of the ratio of two components of the scattered Raman intensities $\frac{\left(\beta_{22}\right)^{2}}{\left(\beta_{33}\right)^{2}}$ from the net-
work theory, single non-Gaussian chain and Gaussian network 
tion for the entire Gaussian range. Note that the network model is initially isotropic; at a stretch of $\lambda=1$ the ratio of the two intensities is 1.0. The same is true for the Gaussian model, although the single non-Gaussian chain is not isotropic. While it may be convenient to describe the orientation in some applications of primarily uniaxial alignment using a single chain for the network polarizability, for example in polymer fluid flows, Fig. 4 demonstrates that the network theory provides a more accurate description of the 3-D amorphous polymer response. Such a description is required when orientation under a general, non-homogeneous deformation is sought. The ability of the network theory to predict the optical anisotropy under a large, non-homogeneous deformation is considered in the following Sections.

\section{Experimental procedures}

\subsection{Materials}

Uniaxial compression and shear tests were conducted on PDMS cubes $(M c=2600 \mathrm{~g} / \mathrm{mol})$ each initially $3.8 \mathrm{~mm}$ high with $4 \mathrm{~mm}$ by $4 \mathrm{~mm}$ bases. Uniaxial compression and shear tests were also conducted using PDMS specimens $(M c=21500 \mathrm{~g} / \mathrm{mol})$ each initially $6.0 \mathrm{~mm}$ on each side.

\subsection{Compression deformation}

Uniaxial compression tests were conducted using an MTS-810 servohydraulic testing frame equipped with dual active actuators. During large uniaxial deformation tests the upper and lower actuators of the MTS platens were driven in synchronization to fix the specimen center in relation to the experimental frame and assure constant optical access to the specimen center. A $2 \mathrm{~mW}$ He-Ne laser was mounted fixed to the experimental reference frame and focussed for normal incidence at the specimen center. The MTS platens were lubricated slightly to insure homogeneous deformation in compression and allow the specimen to expand freely normal to the axis of compression. Quasistatic tests to stretches of $\left(0.3<\frac{l}{l_{0}}\right)$ were conducted. Load and axial displacement were measured directly via a 10lb load cell and an LVDT, respectively.

\subsection{Shear deformation}

The large deformation shear experiments were conducted using a shearing apparatus developed especially for these tests. Figure 5 shows the shear apparatus consisting of an adjustableheight stationary base topped by a fixed-height flat plate supported on smooth bearings. Shear deformations were produced by affixing the specimen between the top plate and the base using an adhesive, then applying a horizontal load to the top plate to draw it along the bearings. Care was taken to insure that initially the top plate produced no load on the specimen. Displacement was measured using a non-contacting LVDT position sensor. Displacement was accomplished via a screw driven actuation system which contained an in-line load cell for load measurement. Friction in the bearings and pulley was negligible. Deformations of up to $\frac{k}{h}=1.1$ were produced. 




Fig. 5. Sketch of the shear test apparatus showing the bearings, adjustable base, specimen, and position and load sensors

Due to specimen incompressibility, the curvature along the unconstrained shearing faces caused inhomogeneity in the deformed specimens. Material at the edges tended to bunch and gather at the oblique corners forcing a redistribution of the deformation throughout the specimen which contrasts the analytical presupposition of a homogeneous simple shear deformation state. The shear apparatus was designed to accomodate an unobstructed laser path along the shear direction and normal to it (parallel to the out-of-plane direction). For the out-ofplane laser path the beam was focussed for normal incidence at the specimen center. The laser path along the shear direction intersected the specimen at an oblique angle which changed with continued shear deformation due to curvature of the specimen surface.

\subsection{Birefringence measurements}

Experimental birefringence measurements for both experiments were conducted using the Babinet-Soleil method of compensation [1]. A circular polariscope consisting of a polarized light source, two $\frac{\lambda}{4}$ plates, an analyzer, and a compensator was used as in Fig. 6. Placing the specimen in the polarized light's path caused a relative angular phase shift, $\Delta$, in the ordinary and extraordinary components of the light emerging from the polariscope. Relative phase shift or (relative) retardation was measured using the compensator and may be directly related to birefringence by

$\Delta=2 \pi \Delta \eta \frac{\delta}{\lambda}$

where $\lambda$ is the wavelength of the laser, $\delta$ is the laser path length through the specimen, and $\Delta \eta$ is the birefringence due to the specimen. This method calculates a bulk property value and does not preclude variable birefringence throughout the specimen during inhomogeneous deformations. 


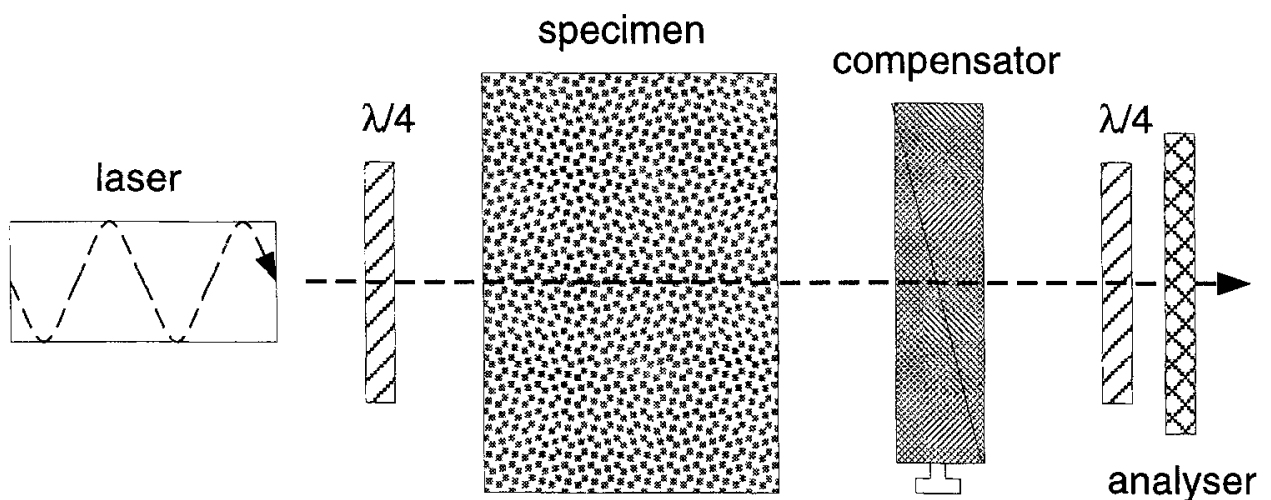

Fig. 6. Circular polariscope schematic showing the laser, quarter wave plates, specimen, compensator, and analyzer

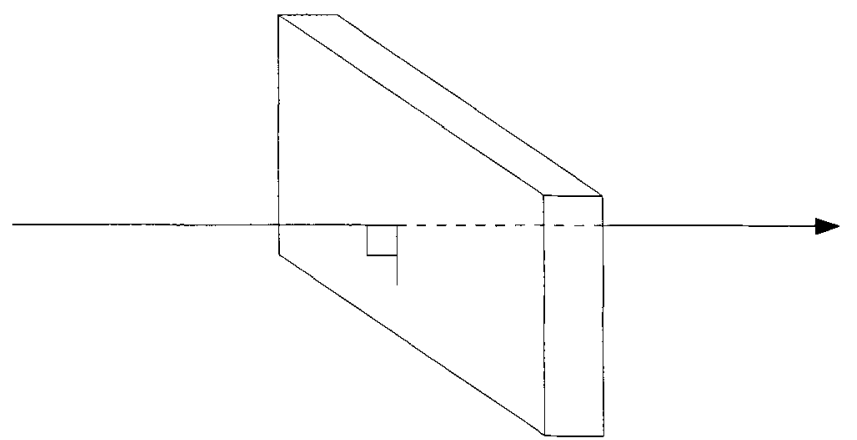

Fig. 7. Schematic describing the plane of interaction as the plane to which the laser path is normal

Data taken from these experiments yielded the principal birefringence in the plane of interaction, or the plane perpendicular to the light's path through the specimen, see Fig. 7. Birefringence measurements were made in two orientations with respect to the shearing deformation and in one orientation during uniaxial tests.

\section{Experimental birefringence determination}

A Cartesian reference frame $(X Y Z)$ and a prinicipal stretch frame ( $x y z)$ are used to facilitate discussion of experimental results. When they differed from the $x y z$ axes, the principal orientations in the plane of interaction are denoted either $\hat{x}, \hat{y}$, or $\hat{z}$. In the plane of interaction, the appropriate $\hat{x}, \hat{y}$, or $\hat{z}$ directions are labelled either $e$ or $o$ for extraordinary or ordinary optic axes, respectively.

\subsection{Compression}

Compression tests were conducted with the laser oriented along the $Z$ axis, see Fig. 8. This $Z$ laser orientation coupled with lack of refraction at the air-specimen interface resulted in an $X-Y$ plane of interaction. The plane of interaction remained parallel to the $X-Y$ plane as the light beam propagated through the specimen for this homogeneous defomation state. Within the plane of interaction the $x(e)$ and $y(o)$ axes lay parallel to the $X-Y$ axes which 


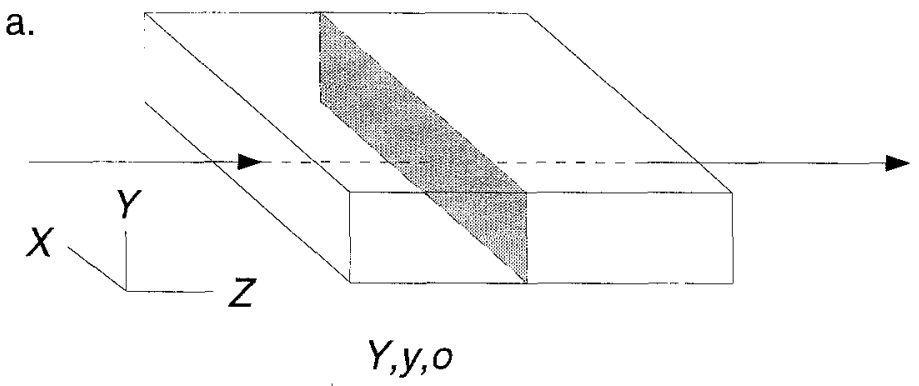

$b$.

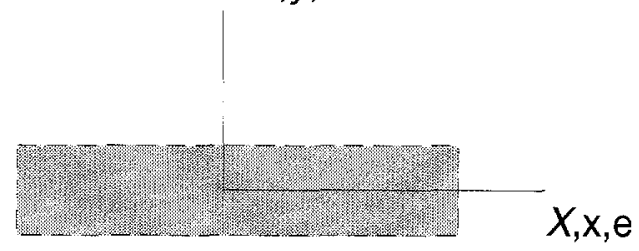

Fig. 8. a Plane of interaction (shaded) with laser in direction $Z, \mathbf{b}$ ordinary $(o)$ and exraordinary $(e)$ principal directions in compression

resulted in the direct measurement of $\Delta \eta_{X-Y}$, see Figs. $8 \mathrm{a}$ and $8 \mathrm{~b}$. Due to symmetry of uniaxial deformation $\Delta \eta_{Y-Z}=\Delta \eta_{X-Y}$.

Measurements in the uniaxial tests were simplified due both to the laser striking normal to the specimen surface, i.e. no refraction at the interface, and to the fixed orientation of the principal stretch directions and principal optic directions, which coincided with the $X Y Z$ axes. This was not the case in the shear experiment because the principal stretch directions rotated with shear deflection and because deformation of the leading edge of the specimen caused refraction of the laser at the air-specimen interface, affecting the light beam path.

\subsection{Large deformation shear}

To fully characterize the birefringence state in the shear experiment, two sets of data were collected simultaneously. The calculation of birefringence for shear experiments conducted using polariscopes aligned along both the $X$ and $Z$ directions is discussed.

\subsection{1 $X$-Axis polariscope}

The $X$-axis polariscope measured the birefringence response in a plane perpendicular to the shear direction. The laser incidence was normal to the specimen surface throughout the deformation such that at the specimen surface the plane of interaction remained parallel to the $Y-Z$ plane during testing, see Fig. 9. The plane of interaction remained parallel to the $Y-Z$ plane as the light beam propagated through the specimen. During shear deformation large rotations of the principal stretch directions occurred. Figure 9 shows the clockwise rotation $\psi$ relating the principal sretch-optic axes in the plane of interaction, $y(o)$ and $z(e)$, to the $Y$ and $Z$ axes. Analysis of the analytical simple shear deformation gradient yields an approximation for $\psi$,

$\psi=\cos ^{-1}\left(\frac{-2}{x \sqrt{x^{2}+4}-\left(x^{2}+4\right)}\right)$,

where $x=\frac{k}{h}$ is the normalized magnitude of shear deflection, $k$ is the shear displacement, and $h$ is the specimen height. Though this presupposes purely homogeneous deformation the assumption gains increasing validity within a region approaching the center of the cube. 
a.

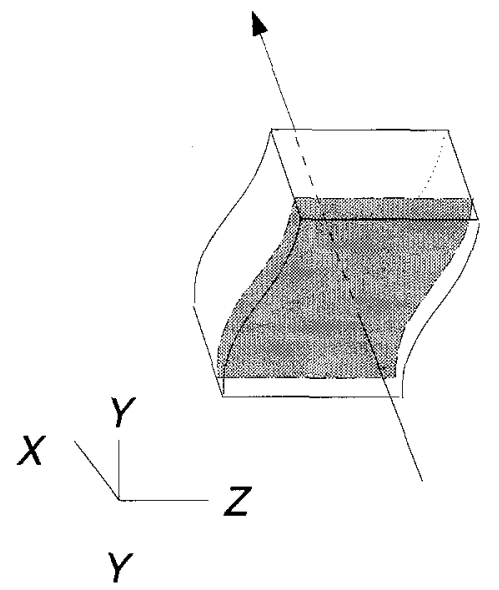

$b$.



Fig. 9. a Plane of interaction (shaded) with laser in direction $X, \mathbf{b}$ ordinary $(o)$ and extraordinary (e) principal directions in shear deformation

Experimental observations indicated that in the $Y-Z$ plane near the specimen center the deformation state approached that of uniform simple shear. The largest sufficiently homogeneous region was smaller than the diameter of the smallest laser beam available, which precluded obtaining an accurate estimation of $\psi$ by Eq. (18). Instead, $\psi$ was determined from a numerical analysis in which $\psi$ was averaged over a volume in the model corresponding to the path of the laser.

The numerical analysis also reported that the deformation through the thickness perpendicular to the shearing direction was homogeneous such that for a given shear deflection the principal directions were constant along the laser path direction which was parallel to the $X$ axis. The plane of interaction remained parallel to the $Y-Z$ plane as the light propagated through the specimen. Experiments in which the polariscope was aligned along the $X$ axis yielded birefringence data $\Delta \eta_{y-z}$ in which the experimental $x y z$ frame was related to the $X Y Z$ frame by the rotation $\psi$.

\subsubsection{Z-Axis polariscope}

An additional set of optical components operating simultaneously and aligned with the $Z$ axis measured the birefringence along the shear direction, see Fig. 10. The deformed specimen edges in shear refracted the incident laser beam. Assuming plane strain deformation which is valid near the specimen center, and making use of Snell's law, the normal to the plane of interaction, i.e. the direction of laser propagation, lay parallel to the $Y Z$ plane and made an angle $\phi$ with the $Z$ axis (see Fig. 10) given by

$\phi=\theta-\sin ^{-1}\left(\frac{\eta_{\text {air }}}{\eta_{0}} \sin (\theta)\right)$ 
a)

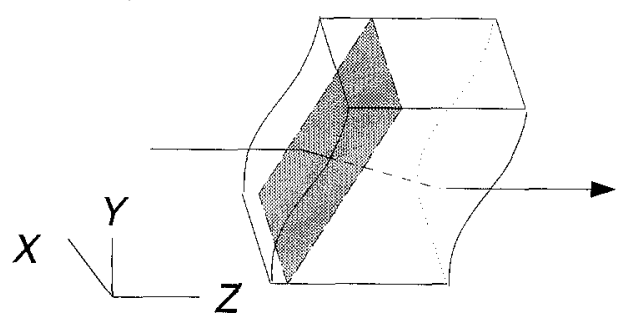

$\hat{y}, e$

c)

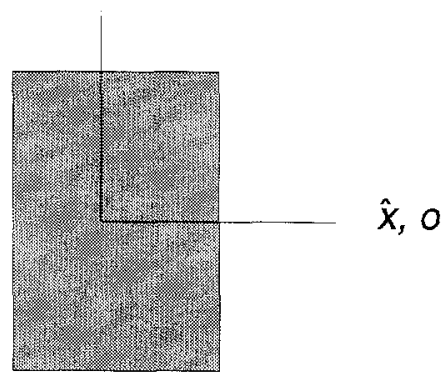

b)

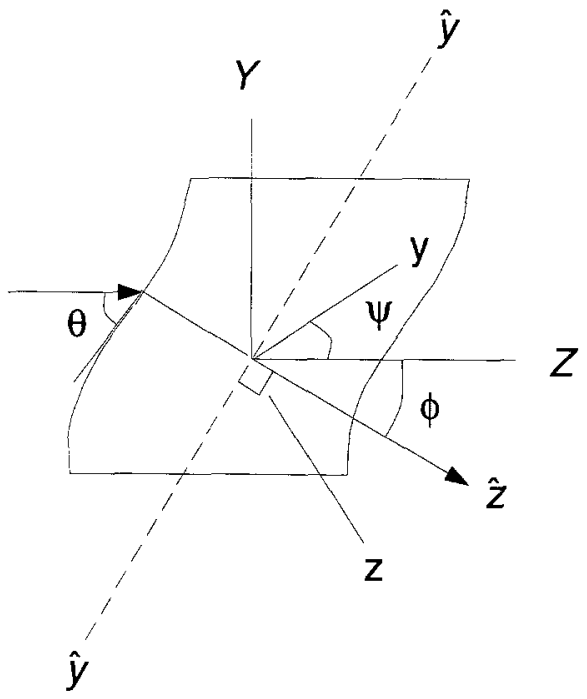

Fig. 10. a Plane of interaction (shaded) with laser in direction $Z, \mathbf{b}$ intersection of plane of interaction $\hat{y}-\hat{y}$ with the $Y Z$ plane, and $\mathbf{c}$ plane of interaction with the ordinary $(o)$ and extraordinary $(e)$ axes

where $\eta_{0}$ is the mean refractive index of the specimen, $\eta_{\text {air }}$ is the refractive index of air, and $\theta$ is the angle of incidence at the air-specimen interface. The refracted laser beam travelled along a path $\hat{z}$, different from $Z$ (and also different from the principal direction $z$ ), but still lay in the $Y-Z$ plane.

The angle $\theta$ can be approximated by its value under purely homogeneous deformation due to the observation that the central portion of the leading edge of the specimen, where the experimenters targeted the incident laser beam, approximates the homogeneously deformed simple shear shape. Numerical results substantiate the assertion that the leading edge of the central portion of the specimen nears the straight-line associated with uniform simple shear. The incident laser beam in the $Z$-axis polariscope was contained within the region of uniform simple shear. The angle $\theta$ is then approximated by

$\theta=\sin ^{-1}\left(\frac{k}{\sqrt{h^{2}+k^{2}}}\right)$.

The principal stretch-optic directions in the plane of interaction $x(e)$ and $\hat{y}(o)$ were related to the $X Y Z$ directions by a rotation $\phi$ about the $X$ axis. The birefringence determined by experiments using the $Z$-axis polariscope yielded $\Delta \eta_{x \hat{y}}$ where the experimental frame $x \hat{y} \hat{z}$ may be related to the $X Y Z$ frame by a rotation $\phi$ about the $X$ axis. Equation (20) clearly shows the dependence of the direction of light propagation on the magnitude of shear deformation.

\section{Numerical analysis}

\subsection{Finite element analysis of deformation}

Finite element modelling of the compression and shear experiments was performed using ABAQUS, a commercial finite element package, in order to simulate the load and stretch results. Three dimensional finite element models, sized to the length, width and height of the specimens, were generated using 1331 three-dimensional linear, hybrid, solid elements, 

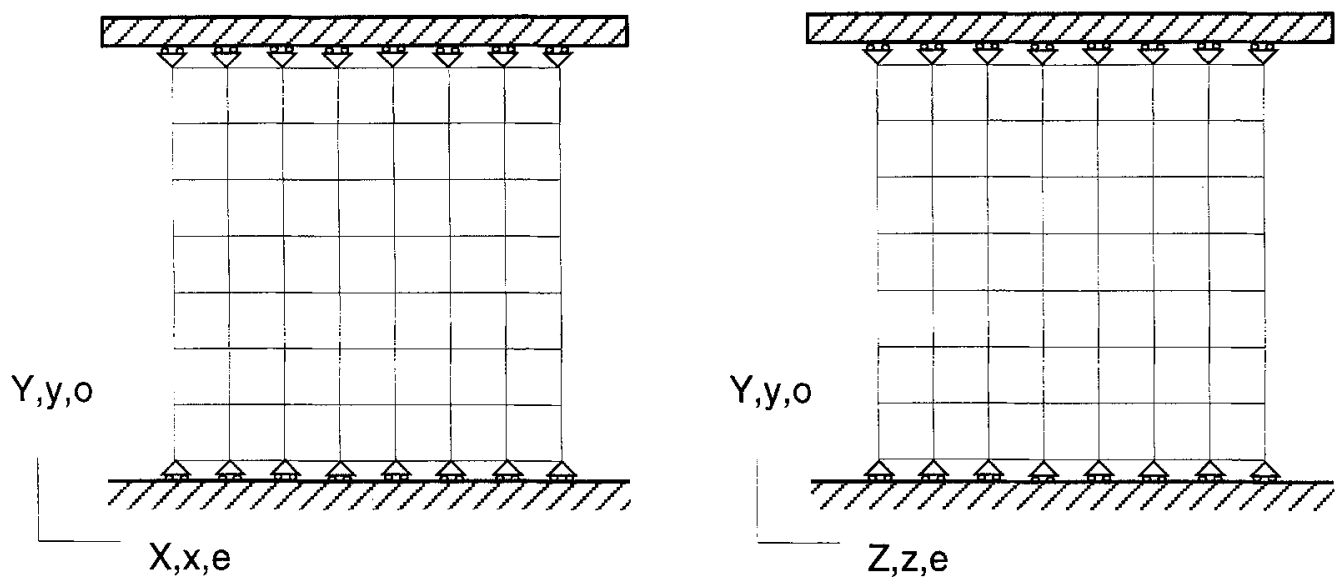

Fig. 11. Finite element model used for the compression simulations

ABAQUS type C3D8H. These elements are capable of linear displacement and constant pressure interpolation. Descriptions of the models, boundary conditions, and imposed deformations are given below.

\subsubsection{Compression}

Figure 11 shows the undeformed model used for simulation of compression experiments. The exposed nodes on the top and bottom faces were allowed to expand freely parallel to the $X-Z$ plane but were constrained to move as a plane in the $Y$ direction. The specimen elements expanded normal to the axis of compression as the top face's free nodes were displaced downward and the bottom face's free nodes remained fixed in the $Y$-direction. The hyperelastic formulation in ABAQUS, which required definition of a strain energy function, was used to generate the finite element displacement solution. An external subroutine was written to calculate the principal birefringences at each integration point based on the deformed shape using Eq. (6). The strain energy density of the eight chain model [31] was used in the simulations,

$$
\begin{aligned}
W= & n k \Theta\left[\frac{1}{2}\left(I_{1}-3\right)+\frac{1}{20 N}\left(I_{1}^{2}-9\right)+\frac{11}{1050 N^{2}}\left(I_{1}^{3}-27\right)+\frac{19}{7000 N^{3}}\left(I_{1}^{4}-81\right)\right. \\
& \left.+\frac{519}{673750 N^{4}}\left(I_{1}^{5}-243\right)+\cdots\right] .
\end{aligned}
$$

The compression simulations were used to characterize the PDMS constitutive behavior. In uniaxial compression, the stretch along the compression axis $\left(\lambda=\frac{l}{l_{0}}\right) \lambda<1$, was uniform throughout the deformation resulting in a uniform true stress $\sigma=\frac{P \lambda}{A_{0}}$ (where we have used incompressibility to relate the current area to the initial area and the current stretch). The compressive true stress versus stretch response was characterized by simulating the compression test and iterating on values of the chain density and limiting extensibility parameters of the eight-chain model, $n$ and $N$, respectively, until they most closely matched experimental results.

\subsubsection{Large deformation shear}

The shear simulation used the model shown in Fig. 12. The free nodes of the bottom row of elements were fixed and the free nodes of the top row of elements were displaced in the shear direction to impose the shear deformation. This top row of nodes was allowed motion in the 

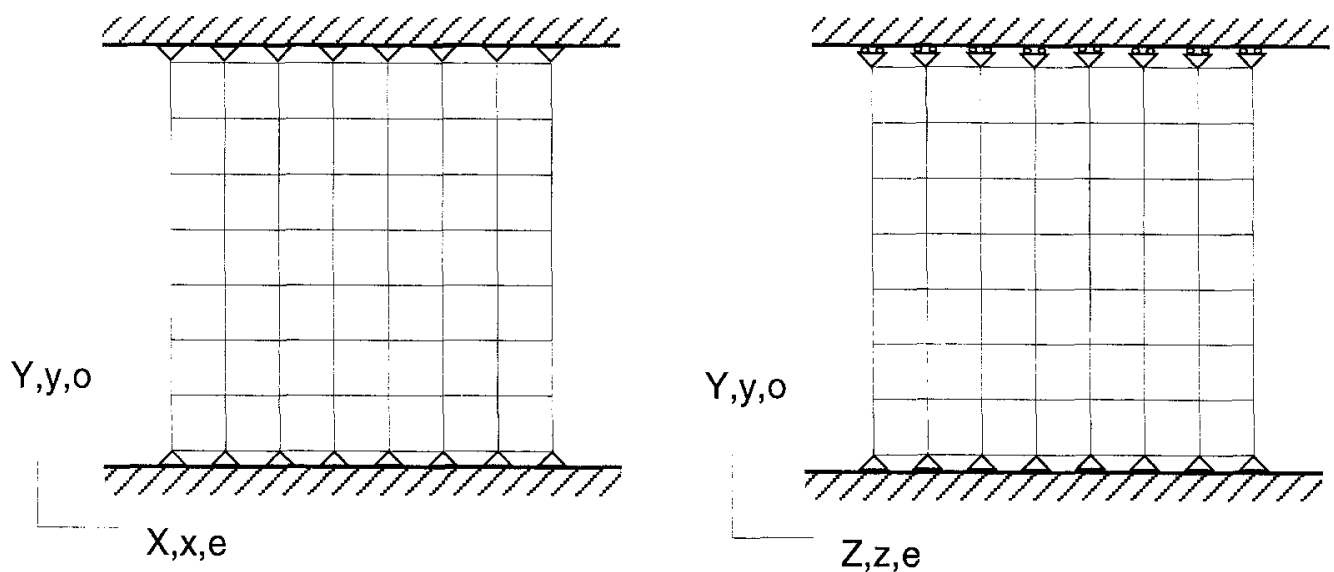

Fig. 12. Finite element model used for the shear simulations

shear direction only and was forced to move as a plane. The eight-chain model was again used as the strain energy function for the hyperelastic specimen elements given the physical parameters found in compression simulations. Shear produced globally inhomogeneous deformation caused by the displacement boundary conditions near the fixed faces. The normalized shear deflections, $\chi$, and load on the top plate were computed for comparison with the experimental results.

\subsection{Finite element analysis of birefringence}

Finite element analysis was used to simulate the birefringence in each experiment. Equation (6) was implemented in a user-defined subroutine to calculate the principal birefringences in the simulations. By projecting a laser path through the simulated specimen corresponding to the path taken through the real specimen, the plane of interaction, local birefringence, and resulting bulk birefringence were calculated numerically.

The following Sections describe the determination of the bulk birefringences from the discrete birefringence values and relate the coordinate frames of the simulations to those of the experiments.

\subsubsection{Compression}

The laser in the experimental setup was aligned along the $Z$ axis. Similarly, the simulation involved a proposed laser along the $Z$ axis, see Fig. 8. Since no refraction occurred at the air specimen interface in the experiment, none was assumed in the simulation; therefore the plane of interaction lay in the $X Y$ plane, see Fig. 8. The $X Y$ plane was the plane of interaction throughout the path of the proposed laser beam. In the compression experiment and in the simulation the principal axes $x y z$ lay parallel to the $X Y Z$ axes making the principal optic axes in the plane of interaction, $e$ and $o$, correspond with $X$ and $Y$, respectively, see Fig. 8. The result was direct calculation of $\Delta \eta_{X Y}$, the birefringence associated with the $X Y$ axes.

\subsubsection{Large deformation shear}

During simple shear the principal axes rotate with shear deformation. The kinematics in shear are best described beginning with the decomposition of the deformation gradient

$\mathbf{F}=\mathrm{VR}$ 
where $\mathbf{R}$ describes a pure rotation and $\mathbf{V}$ a pure deformation. A shear deflection of magnitude $k$ in the $Y-Z$ plane is described by $\mathbf{F}$,

$\mathbf{F}=\mathbf{I}+k_{\mathbf{Y}} \otimes \mathbf{e}_{\mathbf{Z}}$.

The same deformation may be achieved by replacing the deformation $\mathbf{V}$ with its eigenvalue decomposition

$\mathbf{V}=\mathbf{Q}^{\mathrm{T}} \hat{\mathbf{V}} \mathbf{Q}$

such that

$\mathbf{F}=\mathbf{Q}^{\mathrm{T}} \hat{\mathbf{V}} \mathbf{Q R}$.

In Eq. (25) the eigenvectors $\mathbf{Q}$ represent a pure rotation, and the eigenvalues $\hat{\mathbf{V}}$ represent the principal deformations associated with $\mathbf{V}$. The principal axes of the deformation $x, y$ and $z$ are related to the Cartesian $X, Y$, and $Z$ axes by the tensor rotation $\mathbf{Q R}$. The stress-optic law of Eq. (6) determines birefringence in the $\hat{\mathbf{V}}$ frame. Therefore, its results must be rotated into the undeformed Cartesian frame for comparison with experimental results. This is accomplished by constructing the principal refractive index tensor $\mathbf{N}_{\mathbf{x y z}}$ from the principal birefringences using the relationship $\eta_{0}=\frac{1}{3}\left(\eta_{1}+\eta_{2}+\eta_{3}\right)$ and noting that all off-diagonal terms are zero. The Cartesian refractive tensor $\mathbf{N}_{X Y Z}$ is then determined by the tensor rotation $\mathbf{N}_{X Y Z}=\mathbf{R}^{T} \mathbf{Q}^{T} \mathbf{N}_{\mathbf{x y z}} \mathbf{Q R}$.

The above serves to accentuate the fact that the coordinate frame associated with the eight-chain model (the principal stretch frame) in pure simple shear rotates with shear deformation. However, the deformation associated with the term simple shear is only an approximation of the deformation pattern resulting from the large deformation shear experiments. Therefore investigations of birefringence in shear predicated upon the notion of perfectly homogeneous deformation are inherently limited. Methods are described below for better assessing the orientation of the principal frame with respect to the experimental frame.

In a manner identical to that described for the experiments simulating a laser path along the $X$ axis in shear produced a plane of interaction parallel to the $Y Z$ plane, see Fig. 9. The principal stretch-optic axes in the plane of interaction, $y(o)$ and $z(e)$, were related to the $Y Z$ axes by a rotation $\psi$ about the $X$ axis. The numerical subroutine returned $\Delta \eta_{y z}$ at each integration point where the $x y z$ system was related to the $X Y Z$ by a rotation $\psi$ about the $X$ axis. The orientation angle $\psi$ was taken directly from the numerical analysis. The birefringence simulations returned values of $\Delta \eta_{y z}$ where the $x y z$ coordinate system was related to the $X Y Z$ coordinate system by a rotation $\psi$ about the $X$ axis. The birefringence calculations, $\Delta \eta_{y z}$, are the same as the values measured by experiments.

Figure $10 \mathrm{~b}$ depicts the initial orientation of laser beam and its refraction at the interface in the $Z$-axis polariscope. The angle of refraction was a function of the shear deflection according to Eqs. (19) and (20). Figure 10b also shows the proposed laser path. The simulated laser path along the $Z$ axis in shear produced a plane of interaction whose normal in the direction of propagation lay parallel to the $Y Z$ plane making an angle $\phi$ with the $Z$ axis, see Fig. 10 . The $\hat{y}(o)$ and $x(e)$ axes, the principal (optic) axes in the plane of interaction, are shown in Fig. 10c. The experimentally measured birefringence quantity was associated with the $x \hat{y} \hat{z}$ coordinate frame. After generating the principal refractive index tensor $\mathbf{N}_{x y z}$ from the computed principal birefringences as described in Section 5.2.2, the refractive index tensor associated with the experimental frame $\mathbf{N}_{\mathbf{x} \hat{y} \hat{z}}$ was found from a tensor rotation about the $X$ axis of angle $90-(\phi+\psi)$. This rotation correctly aligns the $y z$ axes with the $\hat{y} \hat{z}$ axes, respectively. 
The birefringence $\Delta \eta_{x \hat{y}}$ associated with this orientation was calculated at each integration point throughout the model. The element shape functions were used to interpolate $\Delta \eta_{x \hat{y}}$ from the integration points to values along the laser path. For the $Z$-axis polariscope $\Delta \eta_{x \hat{y}}$ was not uniform along the path. Bulk birefringence for this anisotropic inhomogeneous medium was computed using

$\frac{d \chi}{d \delta}=-g_{12} \chi^{2}+\left(g_{22}-g_{11}\right) \chi+g_{21}$

to define the trajectory of the light's polarization state as it propagated through the medium [34]. In Eq. (27), $\delta$ is the position along the light path, $g_{i j}=f\left(n_{i j}\right)$ are the $\delta$-dependent components of the differential propagation matrix $\mathbf{G}\left(\mathbf{N}_{\mathbf{x} \hat{y} \hat{z}}(\delta)\right)^{2}$, and the complex variable $\chi$ is the ratio of the magnitudes of the two orthogonal components of the light vector. The refractive indices in the experimental frame, the $\eta_{i j}$, were used to construct the differential propagation matrix needed in Eq. (27).

For an anisotropic but homogeneous optic state along the path the solution to Eq. (27) is given as [34]

$\chi\left(\delta, \chi_{0}\right)=\frac{\left[\beta-\frac{1}{2}\left(g_{11}-g_{22}\right) \tan \beta \delta\right] \chi_{0}+\left[g_{21} \tan \beta \delta\right]}{\left[g_{21} \tan \beta \delta\right] \chi_{0}+\left[\beta+\frac{1}{2}\left(g_{11}-g_{22}\right) \tan \beta \delta\right]}$

where $\beta$ is determined from

$\beta=\left[-\frac{1}{4}\left(g_{11}-g_{22}\right)^{2}-g_{21} g_{12}\right]^{1 / 2}$.

The solution to the homogeneous anisotropic case was used as the basis for a piecewise solution to the inhomogeneous anisotropic case. Equation (28) was used to generate $\chi$ values over a small range $\Delta \delta$ in which $\mathbf{G}\left(\mathbf{N}_{\mathbf{x} \hat{y} \hat{z}}(\delta)\right)$ was assumed constant. The values of the components of $\mathbf{G}\left(\mathbf{N}_{\mathbf{x} \hat{\mathbf{y}} \hat{\mathbf{z}}}(\delta)\right)$ were taken to be their averages over the range $\Delta \delta$. The size of $\Delta \delta$ varied dependent on the amount of variation in $\mathbf{G}\left(\mathbf{N}_{x \hat{y} \hat{z}}(\delta)\right)$ but was kept small enough to ensure $1.0 E-4$ accuracy in Eq. (27).

The phase difference (retardation) in the principal optic axes in the coordinate system associated with $\mathbf{G}\left(\mathbf{N}_{\mathbf{x} \hat{\mathbf{y}} \hat{\mathbf{z}}}(\delta)\right)$ was determined by [34]

$\Delta(\delta)=\operatorname{Arg}(\chi(\delta))$.

The birefringence was then found from Eqs. (17) and (30) as

$\Delta \eta(\delta)=\frac{\operatorname{Arg}(\chi(\delta)) \lambda}{2 \pi \delta}$

The bulk birefringence was taken as the value of Eq. (31) at $\delta$ equal to the thickness of the specimen. Since Eq. (30) is an oscillatory function it was necessary to add multiples of $2 \pi$ for each period to account for the monotonic nature of the phase difference.

\section{Results}

Figures $13 \mathrm{a}$ and $13 \mathrm{~b}$ contain the experimental true stress versus stretch and birefringence versus stretch responses of $M_{c}=2600 \mathrm{~g} / \mathrm{mol}$ PDMS in compression along with the analytical predictions of Eqs. (4) and (6). The simulations used $N=10.89, n=1.42 E+19 \mathrm{~mm}^{-3}$, and

\footnotetext{
${ }^{2} \mathrm{G}$ is computed assuming the material in $\Delta \delta$ acts as an ideal, linear, optical retarder [34].
} 




a

Stretch

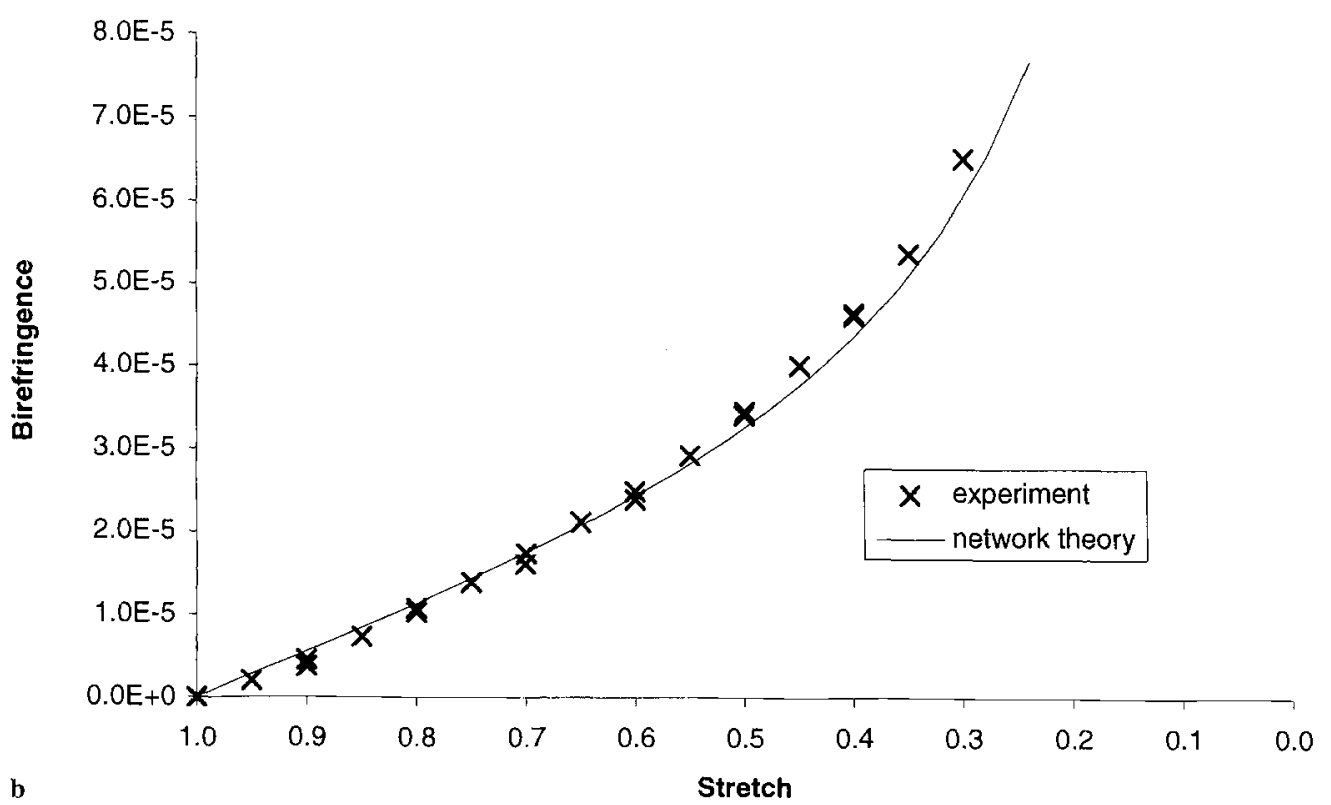

Fig. 13. a True stress versus stretch response, b birefringence versus stretch response of the $M_{c}=2600 \mathrm{~g} / \mathrm{mol}$ PDMS in compression, experimental result and simulation

$\alpha=8.5 E-25$ to capture the entire large deformation responses. The parameters obtained from the compression simulation were used in the finite element simulation of the large deformation shear of $M_{c}=2600 \mathrm{~g} / \mathrm{mol}$ PDMS. The results of the shear analysis appear in Figs. $14 \mathrm{a}$ and $14 \mathrm{~b}$. Figure $14 \mathrm{a}$ contains the force versus normalized shear displacement experimental results and the numerical predictions obtained without adjusting the parameters from the compression analysis. The load data contain some scatter but are well predicted by the theory. Figure $14 \mathrm{~b}$ contains the retardation versus normalized shear deflection data for the 


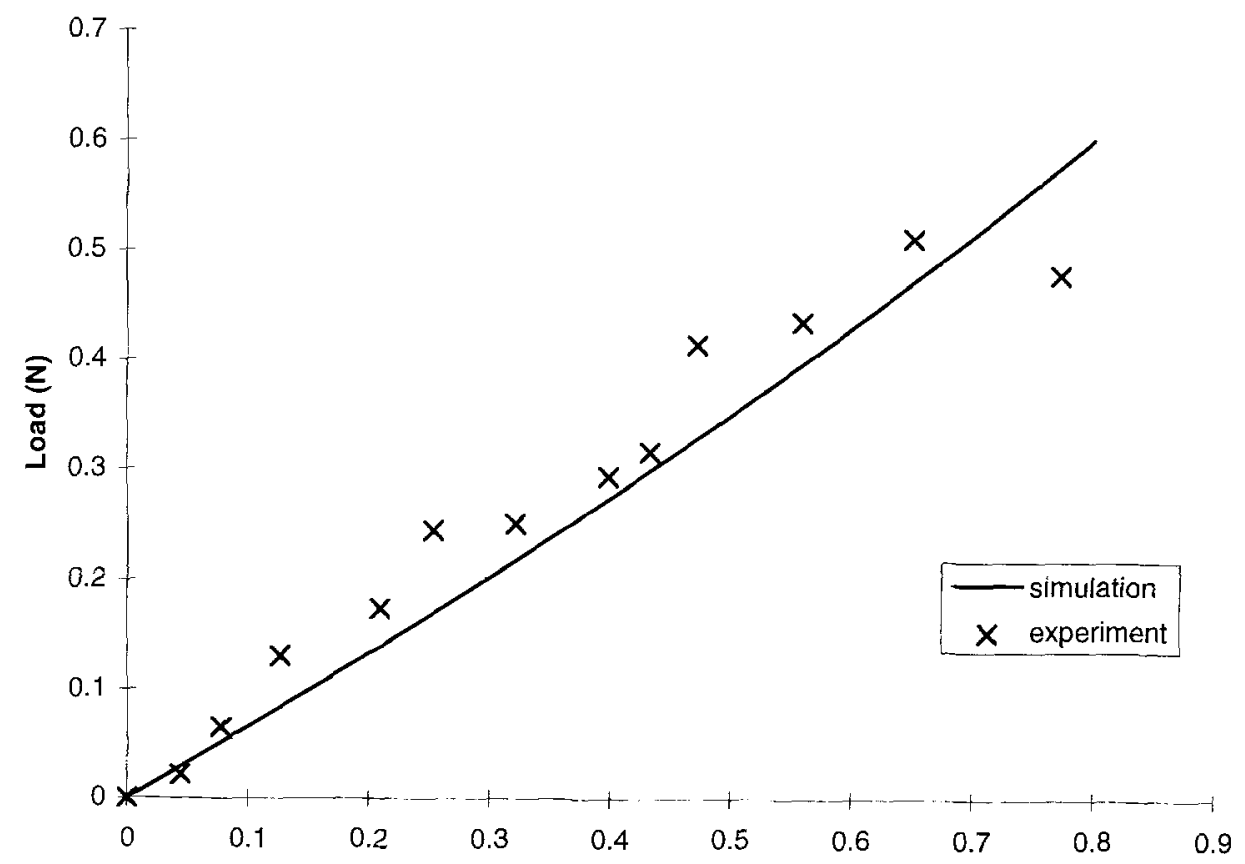

a

Normalized Shear Deflection

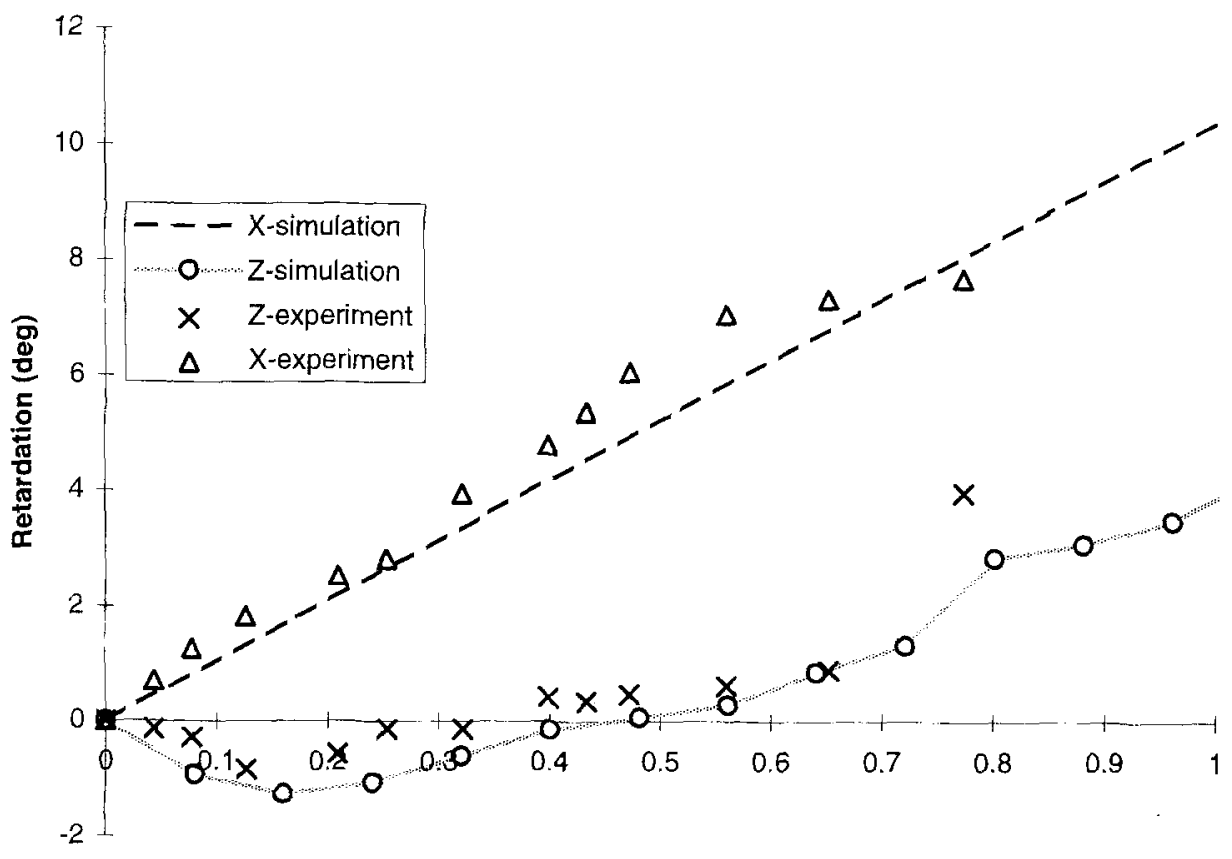

b

\section{Normalized Shear Deflection}

Fig. 14. a Load versus normalized shear displacement, b retardation versus normalized shear displacement for the $M_{c}=2600 \mathrm{~g} / \mathrm{mol}$ PDMS in shear, experimental result and predictions

$X$-axis and $Z$-axis polariscope. The finite element simulations were again obtained without adjusting the material parameters obtained in compression. The theory predicts the experimental results quite well. Particularly, the numerical algorithm used to sum the retardation through the specimen for the $Z$-axis polariscope predicts the initial negative retardation observed in the experiments and the overall shape of the retardation response. 


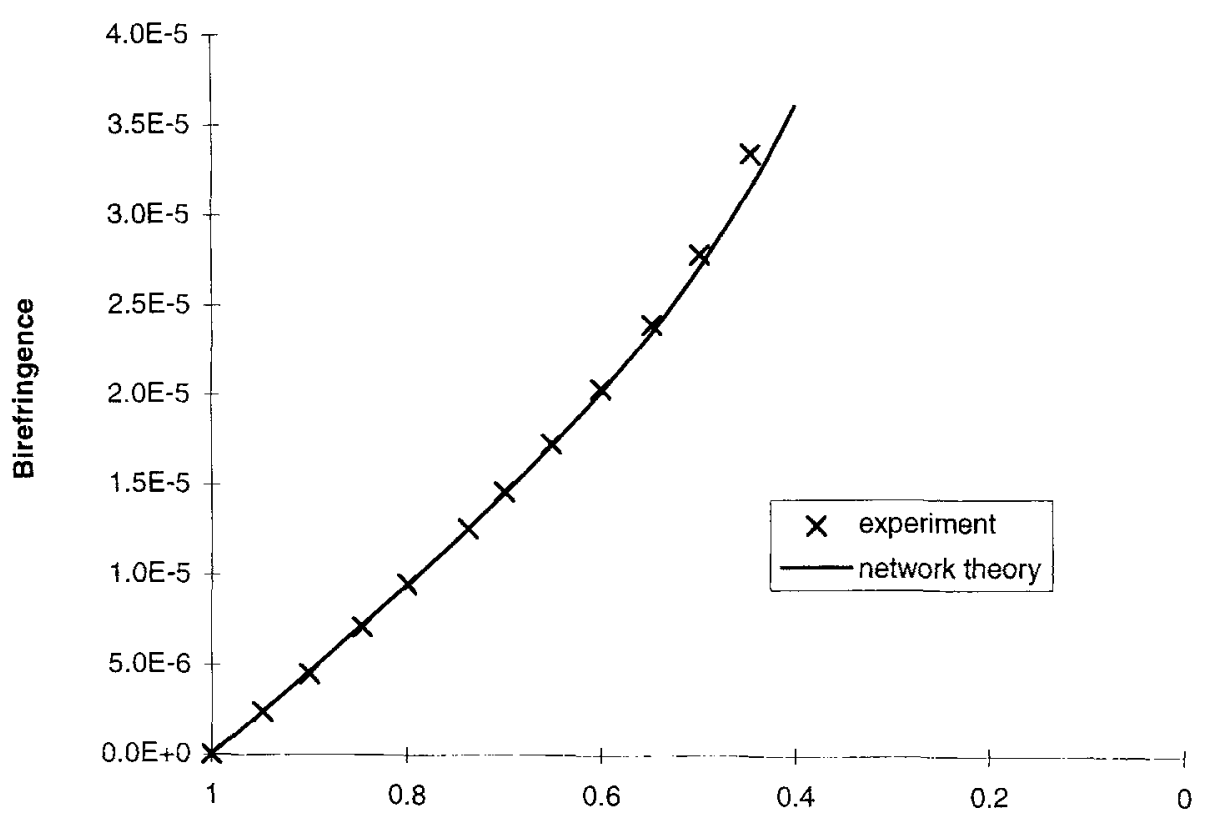

a

Stretch



b

\section{Stretch}

Fig. 15. a True stress versus stretch response, b birefringence versus stretch repsonse of the $M_{c}=21500 \mathrm{~g} / \mathrm{mol}$ PDMS in compression, experimental result and simulation

The analysis was repeated for the true stress versus stretch and birefringence versus stretch responses of $M_{c}=21500 \mathrm{~g} / \mathrm{mol}$ PDMS in compression and shear. The compression results appear in Figs. 15a and 15b; the true stress versus stretch response is simulated in Fig. 15a and the birefringence versus $\lambda$ response in Fig. $15 \mathrm{~b}$ using $N=100 ., n=2.12 E+19 \mathrm{~mm}^{-3}$, and $\alpha=4.66 E-25$. Differences in the values of $\alpha$ for short and long chains are consistent with the 


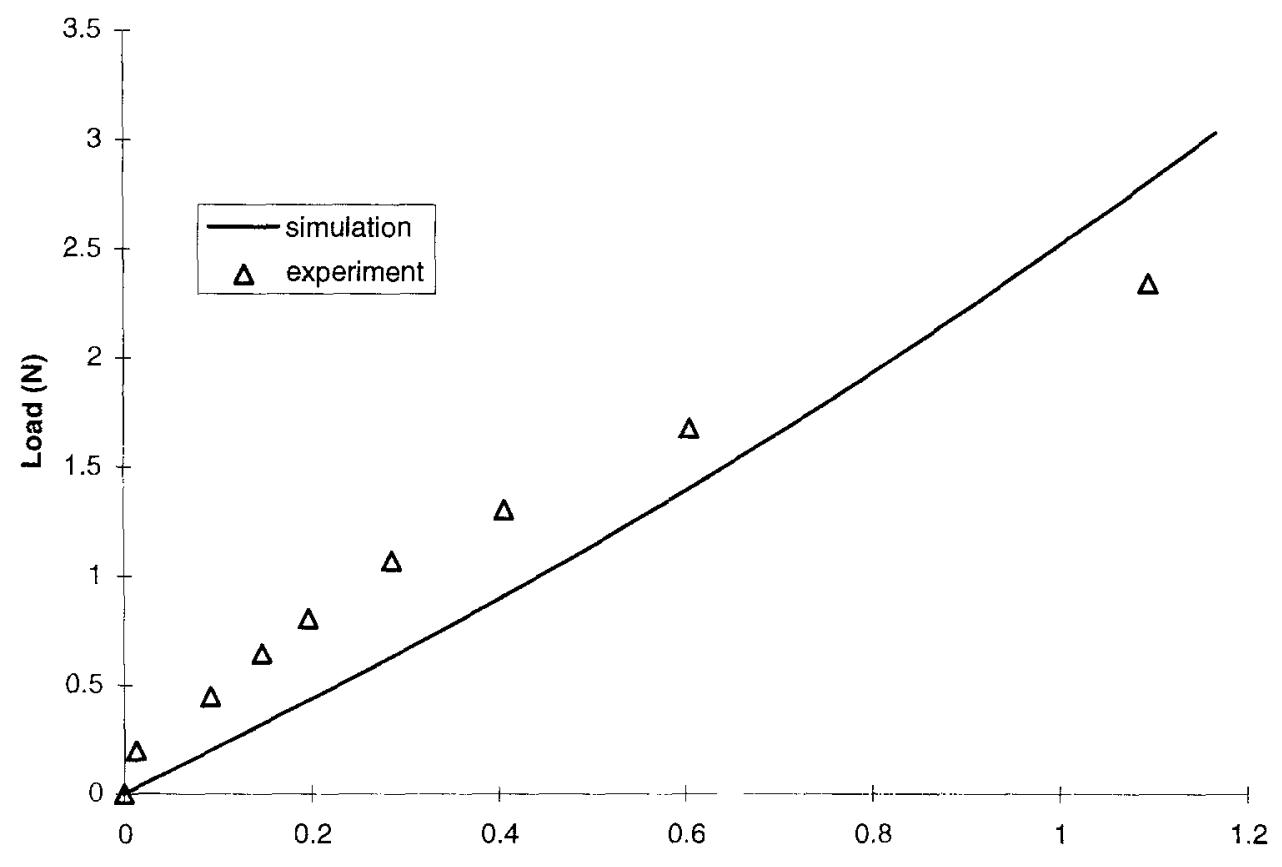

a

Normalized Shear Deflection

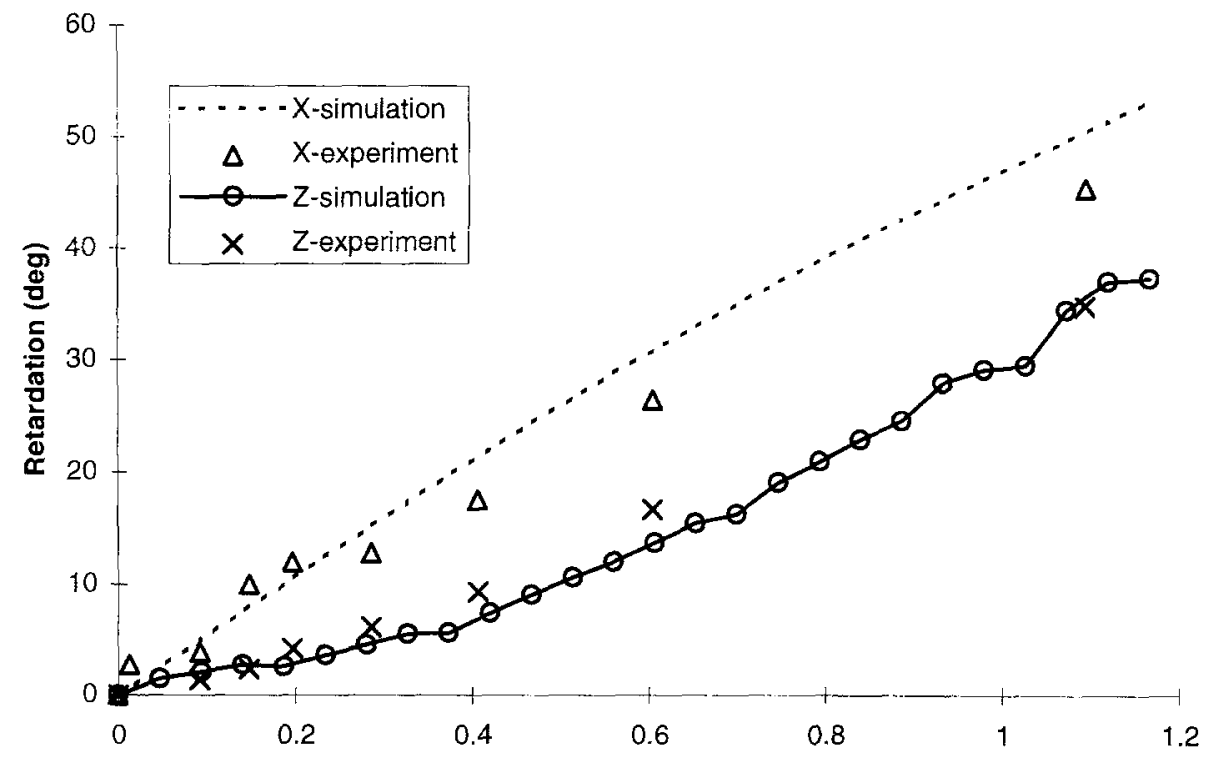

b

Normalized Shear Deflection

Fig. 16. a Load versus normalized shear displacement, b retardation versus normalized shear displacement for the $M_{c}=21500 \mathrm{~g} / \mathrm{mol}$ PDMS in shear, experimental results and predictions

variance of the characteristic ratio, which can vary from $4-12$, with the degree of polymerization for $\mathrm{C}-\mathrm{C}$ backbone bonds [35]. These parameters were used to predict the shear load and retardation responses in Figs. $16 \mathrm{a}$ and $16 \mathrm{~b}$. The simulations predict the data well, especially the retardation measured for both the $X$-axis and $Z$-axis polariscopes. These results demonstrate the ability of the polarizability tensor represented by Eq. (6) to predict the non-homogeneous 


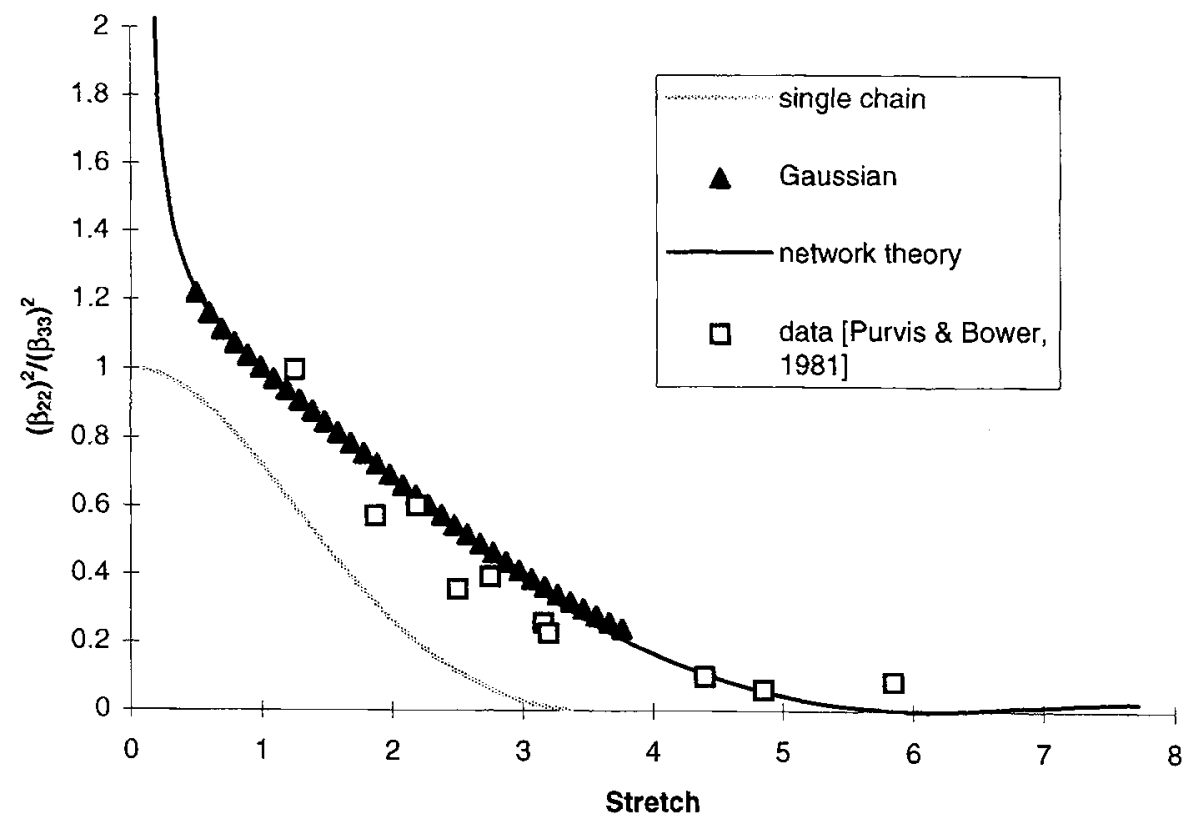

Fig. 17. Ratio of two components of the scattered Raman intensities for PET $\frac{\left(\beta_{22}\right)^{2}}{\left(\beta_{33}\right)^{2}}$ from the network theory, single non-Gaussian chain and Gaussian network. The data are from [17]

shear deformation provided the principal directions of polarization are properly related to the experimental reference frame in which measurement occurred.

The ability of the network theory to predict scattered Raman intensity data was explored using data obtained from axially stretched polyethylene terephthalate (PET) by Purvis and Bower [17]. The $1616 \mathrm{~cm}^{-1}$ line, attributed by many to be a symmetric stretching of carbon bonds in the benzyene rings, is well suited for comparison with the theory. Purvis and Bower measured the $\sum\left(\alpha_{33}^{\prime}\right)^{2}, \sum\left(\alpha_{22}^{\prime}\right)^{2}, \sum\left(\alpha_{12}^{\prime}\right)^{2}$, and $\sum\left(\alpha_{23}^{\prime}\right)^{2}$ intensities for this line versus axial stretch $\lambda$ and reported their data in terms of an orientation parameter $\Phi$ which is proportional to $\left\langle P_{2}(\cos \theta)\right\rangle$ and related to $\lambda$. The network theory predicts $\left(\beta_{12}\right)^{2}=\sum\left(\alpha_{12}^{\prime}\right)^{2}=0$ and $\left(\beta_{23}\right)^{2}=\sum\left(\alpha_{23}^{\prime}\right)^{2}=0$ owing to the symmetry of the contracted network formulation used. In reality these components of the Raman tensor are not zero for the full network response, however the Purvis and Bower results confirm that they are an order of magnitude less than the $\left(\alpha_{33}^{\prime}\right)^{2}$ and $\left(\alpha_{22}^{\prime}\right)^{2}$ intensities. To simulate $\left(\alpha_{33}^{\prime}\right)^{2}$ and $\left(\alpha_{22}^{\prime}\right)^{2}$ we require the parameters $N$ and $\frac{\alpha_{\perp}}{\alpha_{\|}}$in Eq. (13). Purvis and Bower provide $\frac{\alpha_{\perp}}{\alpha_{\|}}=-0.18$ for this vibration, and their reported orientation $\Phi$ data allowed an approximate value of the extensibility in uniaxial tension of $\lambda=7.75$. This results in a chain locking stretch of $\left(\lambda_{c h}\right)_{L}=\sqrt{N}=\sqrt{20}$ or $N=20$. The data of Purvis and Bower are presented in terms of the ratio $\frac{\left(\beta_{22}\right)^{2}}{\left(\beta_{33}\right)^{2}}$ versus axial stretch $\lambda$ in Fig. 17 along with simulations using the network theory of Eq. (13), single non-Gaussian chain and Gaussian network. The results demonstrate that the network theory predicts the anisotropy of Raman scattered intensities very well over the full range of data available. In contrast, the absence of a network description fails to capture either the anisotropy or the network extensibility, as evidenced by the single non-Gaussian chain prediction. 


\section{Concluding remarks}

The network theory developed herein is valid for vibrations that lead to a transversely isotropic description of a link polarizability tensor. The isotropy of the network polarizability depends upon the isotropy of the link, thus it is possible to develop network theories for various link isotropies to explore several Raman lines in a given polymer. Here we have established that a straightforward contraction of the actual amorphous polymer network is sufficient for obtaining useful, valid representations of the optical anisotropy. We have demonstrated the ability to simultaneously predict the constitutive and optical responses in non-homogeneous shearing provided the numerical simulations are properly rotated to the experimental reference frame for comparison. We have used the network theory to predict the scattered Raman intensity versus stretch data of [17] using their published link polarizability ratio and an estimate of the network locking stretch determined from their experiments. This network polarizability theory can be incorporated into constitutive laws for the amorphous phase in semi-crystalline polymers. Orientation in the amorphous phase may be experimentally determined by Raman spectroscopy and compared to simulations in a manner analogous to the current practice of comparing pole figures generated from X-ray scattering data to the texture predictions of polymer crystal plasticity theory. This constitutes a very important step in the verification of semi-crystalline polymer constitutive models as orientation of the amorphous phase has previously not been accessible in a way that allows comparison with simulations.

\section{Acknowledgements}

This work is sponsored by the National Science Foundation (CMS 9410564).

\section{References}

[1] Dally, J. W., Riley, W. F.: Experimental stress analysis. Toronto: McGraw-Hill 1978.

[2] Kannan, M. R., Kornfield, J. A.: Stress-optical manifestations of molecular and microstructural dynamics in complex polymer melts. J. Rheol. 38, 1127-1150 (1994).

[3] Mead, D. W., Larson, R. G.: Rheooptical study of the isotropic solutions of stiff polymers. Macromolecules 23, 2524-2533 (1990).

[4] Mead, D. W., Leal, L. G.: The reptation model with segmental stretch I. Basic equations and general properties. Rheol. Acta 34, 339-359 (1995).

[5] Mead, D. W., Yavich, D., Leal, L. G.: The reptation model with segmental stretch II. Steady flow properties. Rheol. Acta 34, 360-383 (1995).

[6] Pigeon, M., Prod'homme, R. E., Pézolet, M.: Characterization of molecular orientation in polyethylene by Raman spectroscopy. Macromolecules 24, 5687- 5694 (1991).

[7] Galiatsatos, V., Mark, J. E.: Non-Gaussian optical properties of bimodal elastomeric networks. Macromolecules 20, 2631-2632 (1987).

[8] Galiatsatos, V., Subramanian, P. R.: Stress-optical properties of bimodal polymer networks. Macromol. Chem., Macromol. Symp. 76, 233-240 (1993).

[9] Arruda, E. M., Przybylo, P. A.: An investigation into the three-dimensional stress-birefringencestrain relationship in elastomers. Polymer Eng. Sci. 35, 395-402 (1995).

[10] Wu, P. D., van der Giessen, E.: On network descriptions of mechanical and optical properties of rubbers. Phil. Mag. A, 71, 1191-1206 (1995).

[11] Bower, D. I.: Orientation distribution functions for uniaxially oriented polymers. J. Polymer Sci.: Polymer Phys. Ed. 19, $93-107$ (1981). 
[12] Satija, S. K., Wang, C. H.: Polarized Raman scattering studies of chain orientation in hydrostatically extruded polypropylene. J. Chem. Phys. 69, 2739-2744 (1978).

[13] Robinson, M. E. R., Bower, D. I., Maddams, W. F.: Molecular orientation in polyvinyl chloride studied by Raman spectroscopy and birefringence measurements. J. Polymer Sci.: Polymer Phys. Ed. 16, 2115-2138 (1978).

[14] Bower, D. I., Investigation of molecular orientation distributions by polarized Raman seattering and polarized fluorescence. J. Polymer Sci.: Polymer Phys. Ed. 10, 2135-2153 (1972).

[15] Bower, D. I.: Raman scattering from an assembly of partially oriented scatterers. J. Phys. B: Atom. Mol. Phys. 9, 3275-3293 (1976).

[16] Purvis, J., Bower, D. I.: A study of molecular orientation in polymethylmethacrylate by means of laser-Raman spectroscopy. Polymer 15, 645-654 (1974).

[17] Purvis, J., Bower, D. I.: Molecular orientation in polyethylene terephthalate by means of laserRaman spectroscopy. J. Polymer Sci.: Polymer Phys. Ed. 14, 1451 - 1484 (1976).

[18] Jasse, B., Koenig, J. L.: Polarized Raman study of molecular orientation in uniaxially stretched atactic polystyrene. J. Polymer Sci.: Polymer Phys. Ed. 18, 731 - 738 (1980).

[19] Hirsch, J. R., Wang, P. T.: Texture and strength evolution in deformed polypropylene. Text. Microstruct. 13, $101-122$ (1991).

[20] Aboulfaraj, M., G'Sell, C., Ulrich, B., Dahoun, A.: In situ observation of the plastic deformation of polypropylene spherulites under uniaxial tension and simple shear in the scanning electron microscope. Polymer 36, 731 - 742 (1995).

[21] Arruda, E. M., Ahzi, S., Li, Y., Ganesan, A.: Rate dependent deformation of semi-crystalline polypropylene near room temperature. ASME J. Eng. Mat. Technol. 119, 216-222 (1997).

[22] Lin, L., Argon, A. S., Review: structure and plastic deformation of polyethylene. J. Mat. Sci. 29, 294-323 (1994).

[23] Lin, L., Argon, A. S.: Rate mechanism of plasticity in the crystalline component of semicrystalline nylon 6. Macromolecules 27, $6903-6914$ (1994).

[24] Bartczak, Z., Galeski, A., Argon, A. S., Cohen, R. E.: On the plastic deformation of the amorphous component in semicrystalline polymers. Polymer 37, 2113-2123 (1996).

[25] Lee, B. J., Parks, D. M., Ahzi, S.: Micromechanical modelling of large plastic deformation and texture evolution in semi-crystalline polymers. J. Mech. Phys. Solids 41, $1651-1687$ (1993).

[26] Kuhn, W., Grun, F.: Beziehungen zwischen elastischen Konstanten und Dehnungsdoppelbrechung hochelastischer Stoffe. Kolloid Z. 101, 248 -271 (1942).

[27] Wang, M. C., Guth, E. J.: Statistical theory of networks of non-Gaussian flexible chains. J. Chem. Phys. 20, 1144-1157 (1952).

[28] Flory, P. J., Rehner Jr., J.: Statistical mechanics of cross-linked polymer networks. J. Chem. Phys. 11, $512-520(1943)$.

[29] Treloar, L. R. G.: The physics of rubber elasticity. Oxford: Oxford University Press 1975.

[30] Mead, D. W., Harris, C. K., Killough, P. M.: Kuhn-Grun analysis of polarized Rayleigh and Raman scattering experiments to deduce segmental orientation in polymeric systems (submitted).

[31] Arruda, E. M., Boyce, M. C.: A three-dimensional constitutive model for the large stretch behavior of rubber elastic materials. J. Mech. Phys. Solids 41, 389-412 (1993).

[32] Nagai, K.: Photoelastic property of cross-linked amorphous polyethylene. J. Chem. Phys. 40, $2818-2826$ (1964).

[33] von Lockette, P. R., Arruda, E. M.: Network model predictions for the Raman scattered intensities versus deformation for PDMS. In preparation, 1997.

[34] Azzam, R. M. A., Bashara, N. M.: Ellipsometry and polarized light. New Yok: Elsevier 1996.

[35] Strobl, G. R.: The physics of polymers: concept for understanding their structures and behavior. New York: Springer 1996.

Authors' addresses: P. R. von Lockette, Mechanical Engineering and Applied Mechanics, and E. M. Arruda, Mechanical Engineering and Applied Mechanics and Macromolecular Science and Engineering, The University of Michigan, Ann Arbor, MI 48109-2125, U.S.A. 